Working Paper in Economics No. 792

\title{
The effect of information on market activity; evidence from vehicle recalls
}

Inge van den Bijgaart and Davide Cerruti

Department of Economics, October 2020 


\title{
The effect of information on market activity; evidence from vehicle recalls*
}

\author{
Inge van den Bijgaart ${ }^{\dagger}$ and Davide Cerruti ${ }^{\ddagger}$
}

October 7, 2020

\begin{abstract}
We evaluate the effect of vehicle recalls on vehicle transactions in the second-hand market. Using a rich dataset of Dutch vehicle registrations, we exploit the quasi-experimental variation in recalls across nearly-identical cars. We find strong heterogeneities across market segment: vehicles with a lower (higher) list price or some (zero) defects experience an increase (decrease) in transactions after a recall. Based on our theoretical model, this suggests that recalls increase sorting in low-end markets, yet exacerbate adverse selection in high-end markets. Our results shed light on the effect of information arrival in markets subject to uncertainty and information asymmetries.
\end{abstract}

JEL Classification: D12, L15, L62

Keywords: Vehicles, recalls, adverse selection, sorting, information

*We thank Anna Bindler, Claudio Daminato, Massimo Filippini, Reyer Gerlagh, Carsten Helm, Cristian Huse, Jonathan Peterson, Henry Schneider and numerous conference and seminar participants for helpful comments and suggestions. All the errors are our own.

${ }^{\dagger}$ Department of Economics, University of Gothenburg.

${ }_{\ddagger}^{\ddagger}$ Centre for Energy Policy and Economics, ETH Zürich. 


\section{Introduction}

Information availability about product characteristics plays a fundamental role in the correct functioning of markets. In particular, purchasing decisions about expensive durable goods, such as vehicles or household appliances, involve complex considerations about product quality, which is information not always known in advance, or easily observed, by consumers.

Uncertainty about product quality can be mitigated by measures such as quality certifications and mandatory disclosures. A particular type of information disclosure are product recalls due to safety reasons. Recall procedures are in place for a wide range of products, including food, chemical products, textiles, electronics, machinery, furniture, cosmetics, toys, and motor vehicles. For durable goods such as cars, where the defect typically involves one specific component, the product is usually not replaced or reimbursed, but instead repaired for free.

In the automotive market, vehicle recalls are often considered an adverse signal of vehicle quality. Recalls of passenger car vehicles are also extremely common. In 2019 alone, the US National Highway Traffic Association registered 743 recall episodes due to vehicle defects, which affected over 34 million vehicles (NHTSA, 2019), while the European Commission recorded 510 vehicle recall occurrences in the EU, each of which often involved multiple models (European Commission, 2020). Though most recall episodes are not widely reported upon, the largest ones generate substantial media attention. Prominent examples are the Volkswagen emission and Takata airbag scandals, which jointly led to the recall of over 50 million vehicles worldwide.

These recalls create concerns with owners of the affected vehicles, regarding the reliability of their vehicle, as well as its potential resale value.1 Yet, despite their widespread occurrence, the effect of recalls on the recalled vehicle and the used-vehicle markets has been strikingly understudied. Analyzing the effect of recalls on the used-vehicle market is particularly relevant as for many households a car is the second most expensive durable owned. These cars are actively traded, with the used-vehicle market substantially exceeding the primary market in volume. ${ }^{2}$

\footnotetext{
${ }^{1}$ For instance, both Takata and Volkswagen faced class action lawsuits in several countries. For the Volkswagen class action, claimants were seeking compensation for being misinformed about vehicle specifications and approval.

${ }^{2}$ In the US, the volume of trade in the used vehicle market exceeds the new vehicle market by a factor of 2.5 (Cox
} 
In this paper we use a difference in differences methodology to assess the effect of recalls on the used car market, focusing primarily on resale rates. We additionally consider the effect of recalls on the depreciation factor (defined as the ratio of used vehicle asking price to new vehicle list price) of vehicles offered in the used car market. Due to its unexpected nature, a recall constitutes an information shock to both sides of the market. As such, the effect of the recall on the used car market offers an insight into the effect of information arrival in markets subject to substantial uncertainty and information asymmetries.

For this empirical assessment we consider vehicle recalls in the context of the Dutch used car market. In the Netherlands, detailed vehicle-level information on recalls is readily available to, and commonly accessed by, both buyers and sellers. Owners of a recalled vehicle are informed of the recall by letter, while several government and commercial search engines offer information about current and/or past recalls of specific vehicles to prospective buyers. The vast majority of recalls are due to mechanical problems that impose serious security risks for the driver or passengers. Still, while in the Netherlands a recalled vehicle can be repaired free of charge, owners have no obligation to do so, and may continue to use or sell their vehicle without taking any action.

As we show using a stylized model of the used car market, the effect of an adverse signal about vehicle quality on resale rates is not immediate. The model captures two primary motivations for trade in the used car market. The first is sorting, which is driven by differences in the willingness to pay for quality across consumers. Consumers who put a high value on quality will tend to buy new vehicles, and act as sellers on the market for used vehicles. They sell to consumers with a low valuation for quality, who prefer the cheaper, lower-quality, used vehicles over the more expensive new cars. The second motivation for trade is adverse selection: used vehicle sellers have private information about quality, which makes them more inclined to sell especially if this private information indicates that the vehicle quality is low.

The arrival of negative information about vehicle quality affects vehicle transactions through the same two channels. First, the information shock can lead to increased sorting; vehicle sellers Automotive, 2018). In the Netherlands, this is approximately a factor of 4 (CBS, 2019). 
value quality more than buyers, and will thus be more inclined to sell. As such, the arrival of information allows for an improved match of the vehicle to a specific user. Second, the novel information may affect the information asymmetry between buyer and seller. Insofar a recall leads the buyer to downward-adjust its expectation of the privately-observed quality, it will reduce the likelihood of a transaction taking place. Taken together, our theoretical framework points out that the effect of an information shock on trade is ex ante ambiguous; our empirical analysis will provide insight in to which mechanism dominates.

Our analysis uses the Dutch vehicle registration database, which includes detailed vehicle-level information on vehicle characteristics, registration changes and recalls for the entire Dutch vehicle fleet of about 9 million vehicles. The precise classification of the vehicles in our database allows us to adopt an identification strategy that relies on a difference-in-differences strategy exploiting the quasi-experimental variation in the occurrence of recalls within groups of almost identical cars. We group vehicles by version, following official EU regulation for vehicle classification. By this regulation, all cars belonging to the same version must be identical in terms of manufactures, chassis, engine, body style and other fundamental characteristics; only differences in minor features such as color or options are allowed. Yet, while vehicles within a version are virtually identical in terms of observable characteristics, they might still exhibit unobservable differences in the assembly process, such as a different production cycle, plant location, or supplier of the vehicle's components. For this reason, recalls are issued at the vehicle level, and for a specific version, only a subset of vehicles may be recalled. This feature, in combination with a large sample of vehicles, allow us to control for unobserved vehicle characteristics at the version level. Additionally, we are also able to control for unobserved characteristics of recalled cars of a specific version, and for unobserved version-specific time trends.

We find that during the first 6 months from the announcement of a recall, the vehicle resale probability decreases, while, depending on the specification, it increases or remains stable afterwards. The effect over the entire sample period is negative, but not statistically significant across all specifications. When considering different market segments, we identify substantial hetero- 
geneity in the response to vehicle recalls. Vehicles in the high-end used-vehicle market, as proxied by high list price, no past defects, and high vehicle brand reliability, tend to experience a decrease in resales following a recall. This is contrary to the effect for inexpensive and low-reliability vehicles, where we identify an increase in resales due to the recall. Using an ancillary dataset of a large Dutch vehicle marketplace, we do not find an effect of recalls on the depreciation rate of vehicles offered for sale.

Our results are suggestive of recalls affecting the used car market both through increased sorting and a reinforcement of adverse selection. The heterogeneity in our results indicate that for cheap, unreliable vehicles, the sorting effect is dominant. The reduction in transactions of highquality used vehicles however suggests that for those vehicles, the arrival of recall information primarily reinforces adverse selection and thereby need not improve market efficiency.

Our paper is most closely related to Tadelis and Zettelmeyer (2015), who show that in car auctions, the disclosure of information to prospective buyers increases the number of transactions when the information disclosed is new. Contrary to our analysis, Tadelis and Zettelmeyer (2015) only capture the effect of increased information on the demand-side of the market; their experimental design forestalls any supply-side response to the mitigation of the information asymmetry. We instead analyse a situation where the arrival of information is new to both sides of the market, and both parties respond to this new information. In this setting, our analysis highlights that subtle differences in the institutional setting, the market segment, and the type of information revealed can matter greatly for the effect of information on markets.

As highlighted above, despite the common occurrence of vehicle recalls, their effects on the secondary vehicle market remains understudied; we were able to identify only a limited number of contributions, each focusing on the effects of a large, high-profile recall episode. Hartman (1987) find that the 1981-1985 GM safety recalls negatively affected used car prices. Hammond (2013) estimate a negative, albeit small and temporary, effect of the 2009-2010 Toyota safety recall on used-vehicle prices. The more recent work by Strittmatter and Lechner (2020) and Ater and Yosef (2018) explores the effects of the Volkswagen 'dieselgate' scandal on used car markets in Germany 
and Israel, respectively. Strittmatter and Lechner (2020) establish an increase in the supply of Volkswagen diesel vehicles following the scandal, as well as a reduction in the asking price of those vehicles. Ater and Yosef (2018) similarly establish a reduction in resale prices of used Volkswagen vehicles following the scandal, yet identify a reduction in the number of transactions.

Our contribution to this literature is twofold. First, we consider the effect of recalls generally, rather than the effect of a single highly-publicized episode. Second, our empirical approach relies on the quasi-experimental variation in recalls within the same vehicle versions. As such we capture the effect of the recall on the recalled vehicle itself, filtering out any possible spillover effects to other brands or models or to non-recalled cars of the same model, as well as any version-specific trends in resales that might be correlated with the occurrence of a recall. This contrasts past literature which collectively assesses the effect of recalls by adopting a more aggregated approach, comparing across broader vehicle categories such as brands, fuel type, or year of manufacture.

More generally, by shedding light on how consumers may translate information about product safety into market decisions, our paper contributes to a broader literature that evaluates consumer responses to product recalls. Recent contributions to this literature are Freedman, Kearney, and Lederman (2012), who analyze the spillover effect of a series of toy recalls on new toy sales, and Ferrer and Perrone (2017), who perform a detailed assessment of consumer demand responses following a major food safety crisis. Related contributions that consider the effects of recalls on the primary vehicle market are Rhee and Haunschild (2006), Liu and Shankar (2015) and Bachmann, Ehrlich, Fan, and Ruzic (2019). Rhee and Haunschild (2006) and Liu and Shankar (2015) establish that consumers respond more negatively to recalls for brands with a strong quality reputation. Bachmann et al. (2019) consider the Volkswagen scandal. They identify an adverse reputation spillover in response to the scandal, as well as a substitution of new vehicle demand away from diesel and Volkswagen vehicles.

Finally, this paper contributes to the broader literature on the impact of quality certification and information disclosure on consumer choice. Past research in this area considers a variety of sectors, including vehicles (Lewis, 2011), buildings (Eichholtz, Kok, \& Quigley, 2013), appliances (Houde, 
2018), restaurants (Jin \& Leslie, 2003), online marketplaces (Elfenbein, Fisman, \& McManus, 2015), and financial markets (Seira, Elizondo, \& Laguna-Müggenburg, 2017). Our work provides further insight on how information about quality affects market activity.

The remainder of the paper is structured as follows. Section 2 provides the institutional background of the Dutch used vehicle market and recalls. Section 3 puts forward a stylized theoretical framework of information arrival and resale decisions. The data and empirical approach are discussed in Sections 4 and 5, respectively. Results are presented in Section 6, Section 7]concludes.

\section{Institutional background}

This section will briefly set out the institutional context of the Dutch used passenger car market and vehicle recalls.

The used car market in the Netherlands The used car market in the Netherlands is very active: in 2017, out of the 8.2 million cars in the Netherlands, about 2 million changed owner during that year (CBS, 2019). Compared to many other countries, the market is also strikingly transparent. Vehicles are typically advertised in dedicated online marketplaces, where detailed information regarding the main vehicle characteristics is reported. Prospective buyers can also use the vehicle license plate number to obtain detailed information on car characteristics, past inspection results, as well as any recalls. This information is available for free either directly through the search engine of the Netherlands vehicle registration authorities (RDW), or through one of several websites who offer a 'license plate check'. It is common practice to access these resources prior to purchasing a vehicle; the RDW reports that every month, its vehicle registration database is accessed 12-30 million times (RDW, 2018). Following a vehicle transaction, the new vehicle owner is required to register the vehicle in his or her name. A change in ownership can be registered online, or at one of nearly 2000 post offices and car dealerships across the country, and is effective immediately. 
Vehicle recalls As stipulated by EU law, when a common defect which involves the risk of physical injury has been identified, vehicle producers and distributors (henceforth, distributors) have the obligation to notify authorities, inform consumers, recall the product and offer a free repair (European Parliament, 2001; RDW, 2020).

Vehicle recalls are issued at the EU level, but always originate in a single country, typically the one where the vehicles are manufactured. Distributors inform national transport authorities of the recall, who then pass the information on to other EU institutions through the so-called Rapid Alert System. Each national authority then verifies whether any vehicles with the relevant defect are present in their vehicle registry, and if so, initiate the recall procedure. As the Netherlands does not have any major vehicle manufacturers, only very few recalls originate from the Netherlands ${ }^{3}$ Instead, nearly all recalls observed in our data are initiated in other EU countries.

The national vehicle authorities initiate local procedures for handling recalls. In the Netherlands, the relevant authority is the RDW. The RDW supports distributors in contacting and informing the owners of the recalled vehicles about the recall. Due to the active used vehicle market, distributors typically lack accurate data on who owns their vehicles, while the RDW does maintain a detailed registry.

Vehicle owners are informed about a recall by letter $4^{4}$ This letter typically specifies the type of the defect, associated risk, and instructions to arrange an appointment to repair the defective part. The repair is free of charge, yet, there is no requirement for the vehicle to be repaired to remain in circulation or be traded on the used car market 5 Since 2012, in an effort to increase transparency about vehicle recalls, the RDW publishes online (i) a recall registry searchable by vehicle brand and model, (ii) information on open (non-fixed) recalls searchable by license plate, and (iii) a database of vehicle characteristics and current status (open or closed) of each recall by

\footnotetext{
${ }^{3}$ According to the EU Rapid Alert System data (European Commission, 2020), only 11 of the 2694 recall alerts concerning motor vehicles between 2011 and 2019 originated in the Netherlands.

${ }^{4}$ In a small number of recalls, owners are additionally contacted by phone. Newspaper advertisements, as common for e.g., toy recalls, have not occurred in our sample period.

${ }^{5}$ As a consequence, many cars are never repaired. In the Netherlands, this rate is between 10 and 20 percent. Similar, and higher, rates can be found internationally: In the US, for instance, about a third of recalled vehicles remain unrepaired (NHTSA, 2017), while in the UK shares of 47 percent have been reported (Daily Mail,2017).
} 
license plate. The recall information provided typically includes a short description of the defect, the part involved, the potential risk, and the procedures towards receiving the free repair. Using RDW data, several commercial 'license plate check' websites offer vehicle-level information on open recalls free of charge, and in certain cases also closed recalls, either for free or for a small fee. As such, both buyers and sellers have ready access to information about vehicle recalls, and a newly-issued recall can be considered an information shock for all market parties.

\section{Theoretical framework}

To formalize and assess the potential effects of the recall information shock on the secondary vehicle market, this section puts forward a stylized framework of a market for durable goods. The framework builds on the models presented in Hendel and Lizzeri (1999) and Peterson and Schneider (2017). In Hendel and Lizzeri (1999), heterogeneous preferences for quality induces trade in consumer durables, such as cars. Asymmetric information about vehicle quality however creates adverse selection, which reduces, but never eliminates, trade in this market. Peterson and Schneider (2017) build on this approach by considering two dimensions of quality; one symmetrically observed, and one observed only by the seller. They establish a positive relationship between observable quality and the degree of adverse selection, and provide empirical evidence for this result 6

We take a similar approach by assuming that part of the vehicle quality is ex ante unobserved. We consider two types of unobserved quality. First, information about vehicle quality may be asymmetric; it is fully observable to the current vehicle owner, but not to the prospective buyer. Akin to Hendel and Lizzeri (1999) and Peterson and Schneider (2017), this implies that vehicle market features adverse selection. The second type of unobserved quality is symmetric: at the time of trade, neither the current owner nor the vehicle seller can observe its realisation.

This setup allows us to distinguish two potential channels through which information arrival

\footnotetext{
${ }^{6}$ See also Peterson and Schneider (2014) for empirical evidence for the simultaneous presence of sorting and adverse selection in the used car market.
} 
might affect the used car market. For the current vehicle owner, the information shock is 'news' only if it is a credible signal about the symmetrically unobserved part of vehicle quality. The prospective buyer however, might additionally respond to the information shock by updating her expectation of the asymmetrically observed part of vehicle quality.

The specific information shock we consider are vehicle recalls. Vehicle recalls tend to be associated with lower overall vehicle quality:7 We then establish that the effect of a recall on resales depends on which of the channels described above is most important. Specifically, we show that if the recall information shock is primarily a negative signal about fully unobserved quality, it increases the likelihood of a transaction taking place. If instead it is a negative signal about asymmetrically observed quality, this likelihood is weakly reduced.

The model is as follows. We consider a discrete-time economy with infinitely-lived households. Households can choose to consume a durable good, henceforth referred to as a 'car' or 'vehicle'. Per-period utility of a household reads $u=\theta q+z$, where $\theta$ is a preference parameter, $q$ is the quality of the car the households owns, and $z$ is consumption of a numeraire good. Households can consume at most one vehicle each period, and discount the future by a factor $\beta \in(0,1)$. We assume households have heterogeneous preferences for vehicle quality. More specifically, we assume there exists a unit mass of households with a high valuation for quality $\theta_{H}$, and a larger than unit mass of households with low valuation for quality $\theta_{L}$, with $0<\theta_{L}<\theta_{H}$.

Vehicles last for two periods. If a household decides to buy a new vehicle, it uses it for the first period. At the end of the first period, the household must decide to either use the vehicle for one more period, or sell it on the used car market. We assume new cars have homogeneous quality $q^{n}$ and are sold at a constant price $p^{n}$. The quality of a used car is

$$
q^{u}=b+c+d
$$

where $b, c$ and $d$ are random variables distributed according to distributions $f_{B}(b), f_{C}(c)$, and

\footnotetext{
${ }^{7}$ For instance, using US data, automotive research firm iSeeCars established a negative correlation between recall rates and vehicle reliability ratings (iSeeCars, 2018).
} 
$f_{D}(d)$, with support $[\underline{b}, \bar{b}],[\underline{c}, \bar{c}]$ and $[\underline{d}, \bar{d}]$, respectively. We assume that used cars are of positive, albeit lower, quality than old cars: $q^{n}>q^{u}>0.8$

From (1), used vehicle quality can be separated into three components, $b, c$ and $d$. Each component differs in observability. The first component, $b$, is fully observable to both the buyer and the seller. It, for instance, captures verifiable vehicle characteristics, such as model and make, but also mileage and any visible wear and tear. The second component, $c$, is fully observable to the current owner, but not to the buyer. As such, it captures characteristics that can be observed only after a (short) period of use. This includes early prevalence of defects, and also the quality of the engine. The third component, $d$, is realised only at the start of the second period; at the time of vehicle sale, neither the buyer nor the seller observes the value of $d$. We interpret $d$ as capturing any defects or parts of the vehicle quality that are hard to anticipate and will be revealed only after long periods of use, such as a poor gearbox ? $^{9}$

A vehicle recall may reveal information about $c$ and $d$. We assume that the recall, if anything, is considered an adverse signal about vehicle quality; the conditional expectation of $c$ and $d$ is weakly lower in the presence than in the absence of a recall. This is formally expressed by $\mathbb{E}[c \mid \mathrm{I}=1] \leq$ $\mathbb{E}[c \mid \mathrm{I}=0]$ and $\mathbb{E}[d \mid \mathrm{I}=1] \leq \mathbb{E}[d \mid \mathrm{I}=0]$, where $\mathbb{E}[\cdot]$ is the expectations operator and $\mathrm{I}$ is an indicator equal to 1 if the vehicle has been subject to a recall, and zero otherwise ${ }^{10}$ We assume that both buyers and sellers observe this information signal.

Before we proceed to determine the equilibrium in the used car market, we make two additional assumptions regarding the new vehicle price. First, we assume the price is sufficiently low such that high-valuation households derive strictly positive expected benefits from new vehicle ownership. Unless this assumption is satisfied, no households will be willing to purchase new vehicles, and no cars will be used. Second, we assume the price is sufficiently high such that low-valuation households never purchase new vehicles. Jointly this implies we require $p^{n} \in\left(\theta_{L} q^{n}+\beta \theta_{H} \mathbb{E}\left[q^{u}\right], \theta_{H} q^{n}+\beta \theta_{H} \mathbb{E}\left[q^{u}\right]\right)$.

\footnotetext{
${ }^{8} \mathrm{~A}$ sufficient assumption for this is $\bar{b}+\bar{c}+\bar{d}<q^{n}$ and $\underline{b}+\underline{c}+\underline{d}>0$

${ }^{9}$ The gearbox is one of the most expensive vehicle parts to replace. It has a typical lifetime of about $300,000 \mathrm{~km}$, which implies that for many cars, it is never replaced.

${ }^{10}$ The recall might also be correlated with $b$, yet, as $b$ is fully observed, it will not affect $\mathbb{E}[b]$.
} 
For expositional purposes, the remainder of the analysis will assume that the distributions $f_{C}(c)$ and $f_{D}(d)$ are independent of $b$, and that, conditional on I, $c$ and $d$ are independently distributed.

Equilibrium In equilibrium, high-valuation households will purchase new vehicles, while lowvaluation households demand used vehicles. After the first period of use, a high-valuation vehicle owner is willing to sell the vehicle whenever the expected value of continued use is equal to or below the expected value of selling the vehicle and purchasing a new vehicle instead. This is the case if

$$
\theta_{H}(b+c+D(\mathbf{I})) \leq p^{u}+\left[V-p^{n}\right]
$$

where $D(\mathbf{I}) \equiv \mathbb{E}[d \mid \mathbf{I}]$ is the expectation of $d$ conditional on $\mathbf{I}, p^{u}$ is the used vehicle price, and $V$ is the expected welfare derived from purchasing a new vehicle: $V=\theta_{H} q^{n}+$ $\beta \mathbb{E}\left[\max \left\{\theta_{H} q^{u}, p^{u}+\left[V-p^{n}\right]\right\}\right]$. From (2) one directly observes that for a given $p^{u}$, the vehicle owner is more likely willing to sell the lower is the realisation of $c$, and the lower is the expectation of $d$. In equilibrium, the $p^{u}$ is equal to the willingness to pay by the low-valuation households, which is in turn equal to $\theta_{L}$ times expected vehicle quality:

$$
p^{u}=\theta_{L}[b+C(b, \mathbf{I})+D(\mathbf{I})]
$$

where $C(b, \mathrm{I}) \equiv \mathbb{E}[c \mid b, \mathrm{I}]$ is the expectation of $c$ conditional on the realisation of I, and the observation that the seller is willing to offer a car with observable quality $b$ on the market. We can then use (3) to rewrite (2) as

$$
b+c+D(\mathrm{I}) \leq \frac{V-p^{n}}{\theta_{H}-\theta_{L}}+\frac{\theta_{L}}{\theta_{H}-\theta_{L}}(C(b, \mathrm{I})-c)
$$

A vehicle will be sold on the used car market whenever (4) is satisfied ${ }^{11}$ Equation (4) highlights two motives for trade in the used car market. The first motive is sorting. Used vehicle sellers have a higher marginal willingness to pay for quality than buyers. A low expected quality realization

\footnotetext{
${ }^{11}$ Determining the equilibrium on the vehicle market additionally requires solving for $C(b, \mathrm{I})$. In Appendix $\mathrm{B}$ we do so for a specific distribution $f_{C}(c)$.
} 
$b+c+D(\mathrm{I})$, then implies that sellers are more likely willing to sell the vehicle, as this allows them to upgrade to a new vehicle with higher expected quality. The second motive is adverse selection. The buyer's expectation of $c, C(b, \mathrm{I})$, might deviate from its actual value. Whenever the realisation of $c$ is low relative to $C(b, \mathrm{I})$, the actual quality of the vehicle is lower than the buyer's expectation. In this situation the seller will be particularly inclined to sell the vehicle. Of course the converse is also true. If $c$ is high relative to $C(b, \mathrm{I})$, buyers are willing to pay less than the 'true' value of the vehicle, and consequently vehicle owners are less inclined to sell.

A recall will then affect the market through those same two channels. First, a recall may lead both buyers and sellers to downward adjust their expectation of $d$. This is the case whenever $D(1)<D(0)$. Then, the recall information shock will induce increased sorting on the used car market, and correspondingly increase the likelihood that a transaction will take place. The reduction in $D$ following a recall may have an additional, indirect, effect through $C(b, \mathrm{I})$, as the drop in $D$ (I) implies that for given $b$, sellers will be willing to sell vehicles with a higher asymmetrically observed quality $c$. Knowing that higher-level $c$ vehicles are offered on the market for recalled cars, buyers assign a higher expectation of $c$ to such a car: $C(b, 0)<C(b, 1)$. While the drop in $D$ reduces the used-vehicle price (see (3)), this indirect effect on $C(b, \mathrm{I})$ increases the buyer's willingness to pay for the vehicle and thus the vehicle price, thereby reinforcing the positive effect of the recall information on vehicle transactions. All in all, the sorting channel predicts an increase in vehicle transactions following a recall, with an ambiguous net effect on the vehicle price ${ }^{12}$

Second, the recall may directly affect the buyer's expectation of $c, C(b, \mathrm{I})$. More specifically, vehicles with low asymmetrically observed quality may more likely be subject to a recall. In this case $C(b, 0)>C(b, 1)$, and buyers will expect a car with observable quality $b$ to have a lower asymmetrically observed quality $c$ if the car has been subject to a recall. Such a change in expectations following the recall will reduce the buyer's willingness to pay, reduce the vehicle price, and in turn reduce the likelihood that the vehicle is traded on the used car market.

This second channel highlights that in the presence of information asymmetries, the recall may

\footnotetext{
${ }^{12}$ The example in Appendix $B$ shows that the effect of the recall on the used vehicle price can indeed go both ways.
} 
lead to reduced trade in the recalled vehicle. It is relevant to note that those vehicles that are most likely pulled out of the market following a recall are those for which the recall leads to an increase in information asymmetries. This can observed as follows. Consider (4), and suppose that the buyer cannot accurately infer quality $c$; for a given $b$ and I, sellers with different $c$ are active on the market. Suppose also that the average quality $c$ is lower on the market for recalled than nonrecalled vehicles: $C(b, 0)>C(b, 1)$. Then from (4), the sellers closest to the threshold and thus most likely to pull the vehicle off the market are those with the highest level of $c$, i.e., those with $c>C(b, 0)$. Then as $C(b, 0)>C(b, 1)$, the recall increases the information asymmetry between the buyer and 'marginal' seller. ${ }^{13}$

The remainder of the paper focuses on estimating the effect of a recall on the resale likelihood, as well as prices. Equations (3), (4) and subsequent discussion will aid us in interpreting our empirical results. More specifically, it allows us to assess whether the arrival of information in the form of recalls, primarily affects the market by allowing for increased sorting, or that it causes more owners to refrain from selling their vehicles, by affecting underlying information asymmetries.

In our empirical analysis we estimate the effect of recalls both for the market as a whole, as well as different segments of the market. Across segments, the distributions of $c$ and $d$ likely differ, and also the preferences of the market participants, $\theta_{H}$ and $\theta_{L}$ may vary. Equation (4), for instance, suggests that, for a given gap in quality preferences of sellers and buyers $\theta_{H}-\theta_{L}$, the adverse selection channel which operates through $C(b, \mathrm{I})$ and reduces resales is less influential on a market where buyers have a low quality preference $\theta_{L}$. Similarly, a recall may be considered a stronger signal about individual vehicle quality in some segments than others; in the model, this can be captured by a stronger correlation between recalls and low quality $c$ and $d$ for those segments. It is not ex ante clear whether such a signal is likely stronger or weaker for a high-end segment. For instance, a recall may be more surprising to both buyers and sellers if the car has a strong quality reputation. Simultaneously, a strong reputation may mitigate the extent to which market

\footnotetext{
${ }^{13}$ Depending on how 'noisy' the recall information shock is, this increase in information asymmetries may be more or less common. For instance, the marginal vehicle will never be recalled if recalls only affect the lowest quality $c$ vehicles.
} 
participants update the perception of vehicle quality after the arrival of recall information. ${ }^{14}$

Finally, it is important to highlight that the discussion above assumes that a recall is considered an adverse signal of vehicle quality. This is the conventional assumption, and consistent with the negative effects of recalls on new vehicle sales and firm stock market value identified in the literature. ${ }^{15}$ We however cannot rule out that, in some instances, a recall is instead perceived positively. For instance, a well-managed recall may be interpreted as the manufacturer putting care into ensuring continued satisfaction with its customers. If indeed recalls are perceived as a positive signal for vehicle quality, the above theoretical implications are opposite: it would lead to reduced transactions through the sorting channel, and increased transactions through a mitigation of adverse selection.

\section{Data description}

Data sources For our analysis we use registration data collected by RDW. We consider passenger car registration data from November 2017 to March 2019. For each vehicle, we have information on the license plate, ownership changes, date of first registration, and detailed information on a wide range of vehicle characteristics. Additionally, we know the type, variant, and version of each vehicle, which allows us to distinguish between different groups of vehicles belonging to the same brand or model. Vehicles within the same version are identical in the most of vehicle characteristics, with the exception of minor traits (e.g., color) or accessories 16

We complement the registration data with data from the RDW recall registry, which includes all recalls since 2012. For each recall, we know the date the recall was issued by the manufacturer and RDW, and the date of notification of car owners. The registry reports also the number of vehicles recalled in the Netherlands and worldwide, a classification of the risk from the defect

\footnotetext{
${ }^{14}$ There exists evidence that establishing a brand reputation through advertising can mitigate the adverse customer response after 'bad news' (Barrage, Chyn, \& Hastings, 2020). Rhee and Haunschild (2006) however document that high-reputation automakers experience larger declines in market share following a recall.

${ }^{15}$ See e.g., Rhee and Haunschild (2006) and Hammond (2013). Analogously, Beattie, Durante, Knight, and Sen (2020) find that newspapers provide lower coverage of recalls issued by manufacturers that are regular advertisers.

${ }^{10}$ We explain in detail how these categories are determined and their role in our identification strategy in Section 5
} 
(serious, average, low), the vehicle part that is considered defective, and the method through which the recall was communicated to the vehicle owner. Our final dataset is thus in a panel format, where for each vehicle-month we observe whether the car changed owner and whether a recall was issued.

Market share and recalls by manufacturer While the Dutch passenger car fleet includes a wide range of brands, there are six dominant manufacturers: Volkswagen, Opel, Peugeot, Renault, Ford and Toyota $\sqrt{17}$ These manufacturers all have market shares exceeding $6 \%$, and collectively account for about half of the total vehicle fleet (see Figure A.1 in Appendix A). The remaining top 20 brands are primarily Asian and European, with individual market shares between 1\% and 5\%.

Some manufacturers issue more recalls than others. Figure 1 displays for the major manufacturers the share of registered vehicles that has been recalled at least once between November 2017 and March 2019. ${ }^{18}$ We observe that during this period, Mitsubishi and Toyota recalled the greatest proportions of their vehicle fleet, 42 and 32 percent, respectively. These high shares can mostly be attributed to the unusually expansive and continuing Takata-Toyota airbag recall.

Characteristics of vehicle recalls Vehicle recalls are a very common occurrence. From November 2017 to March 2019 we observe 896 recall episodes, which jointly involved 957,572 vehicles. This amounts to about $9.47 \%$ of vehicles ever in circulation during that period. If we additionally consider earlier recalls, we find that $1,957,581$ vehicles, or $19.37 \%$ of the fleet, has been recalled at least once. Appendix A provides additional information about recalls in the Netherlands in terms of severity, part involved, and number of vehicle involved per recall.

The number of recalled vehicles varies noticeably over time (Figure 2), both in absolute terms and as a share of the size of the fleet in that particular month ${ }^{19}$ On average, we observe a monthly

\footnotetext{
${ }^{17}$ In the remainder, we will use the terms "brand" and "manufacturer" interchangeably.

${ }^{18}$ The figure excludes brands with very small market shares. Several of these brands have recall shares exceeding 50 percent. This is probably due to the fact that those manufacturers sell relatively few distinct vehicle models, which implies that a single recall more likely involves a large share of their total fleet.

${ }^{19}$ The size of the fleet changes from month to month due to new registrations (new vehicle sales and used car imports), exports and vehicles that are stolen or scrapped. As a consequence, the number of vehicles ever in circulation, as referred to in the previous paragraph, exceeds the size of the fleet in a given month by around 1/8.
} 
Figure 1: Share of recalls by brand (Nov 2017-Apr 2019), top 20, only brands with $>0.1 \%$ market share

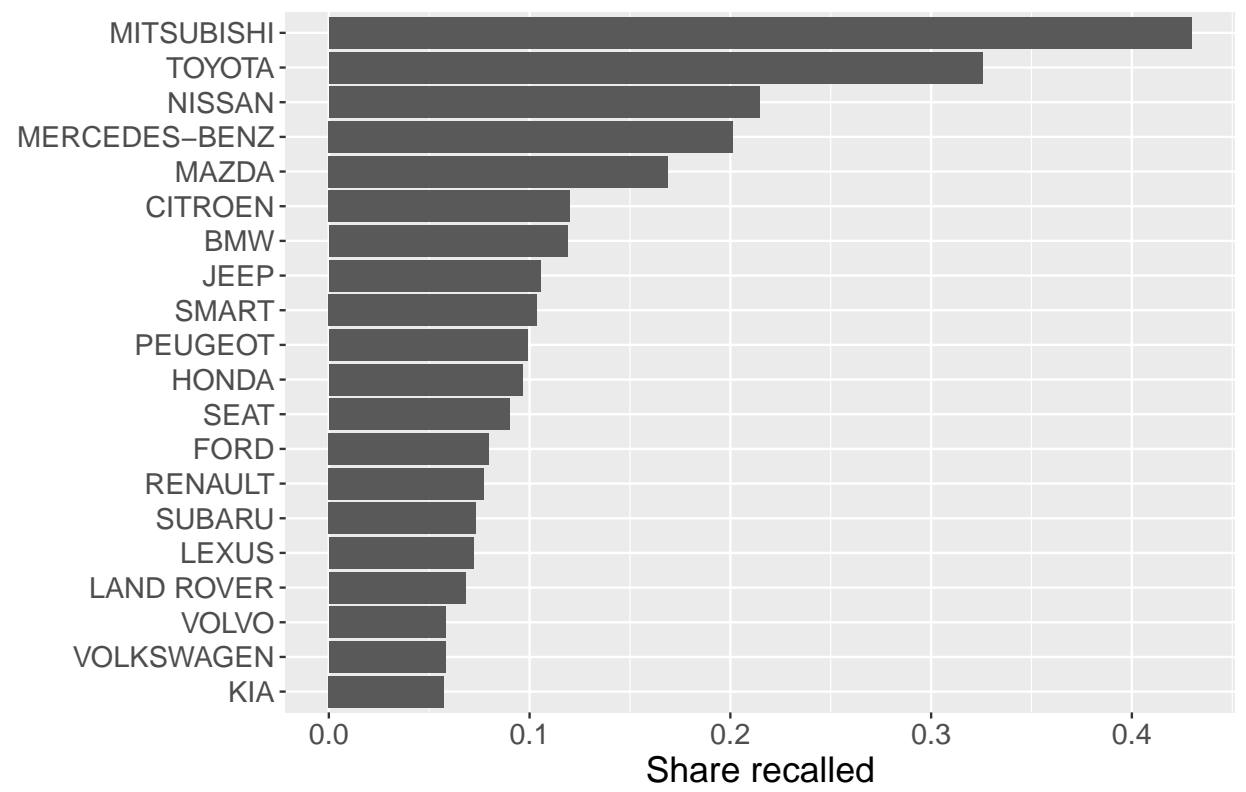

recall rate of about $0.59 \%$, which amounts to about 51,000 vehicles each month.

Vehicle resales In our sample period, ownership changes are frequent: on average $2.78 \%$ of the vehicle fleet changes owner in a given month. In our sample period, we record a total of 4,246,779 ownership changes. 20

Figure 3 shows the share and the total number of resales over time. We observe relatively high resale rates from January 2018 through March 2018. This is likely explained by changes in new vehicle registration taxes becoming effective as of January 2018. Towards the end of our sample period, the rate of monthly resales is fairly stable between $2.5 \%$ and $2.9 \%$

\footnotetext{
${ }^{20}$ While in the text we use the terms ownership changes and resales interchangeably, we cannot verify that each ownership change corresponds to an actual transaction. It is possible that ownership changes very close in time are simply the result of administrative changes within a dealer. In our analysis, we find that our results are robust to excluding ownership changes that occur soon after the previous one ( 3 months, 6 months, 12 months).
} 
Figure 2: Number and share of vehicles recalled per month

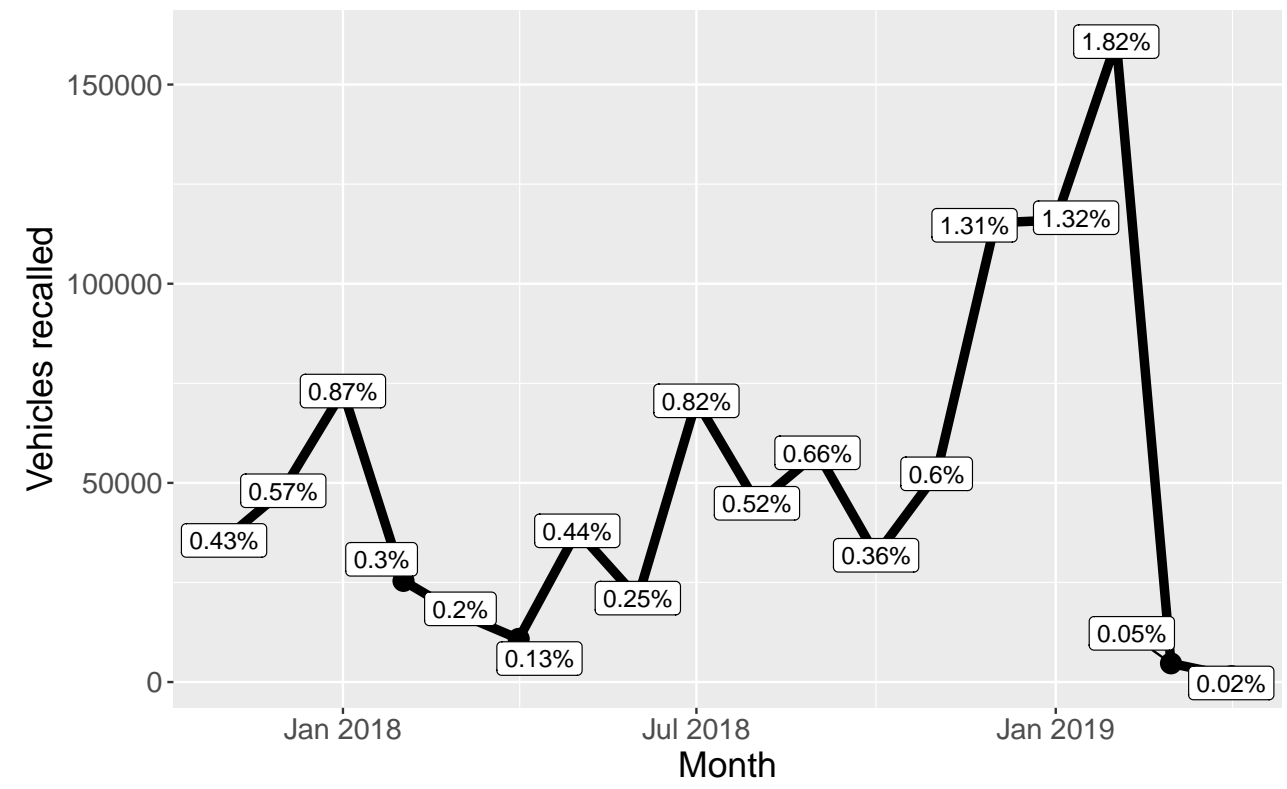

Figure 3: Number and share of vehicles resold per month

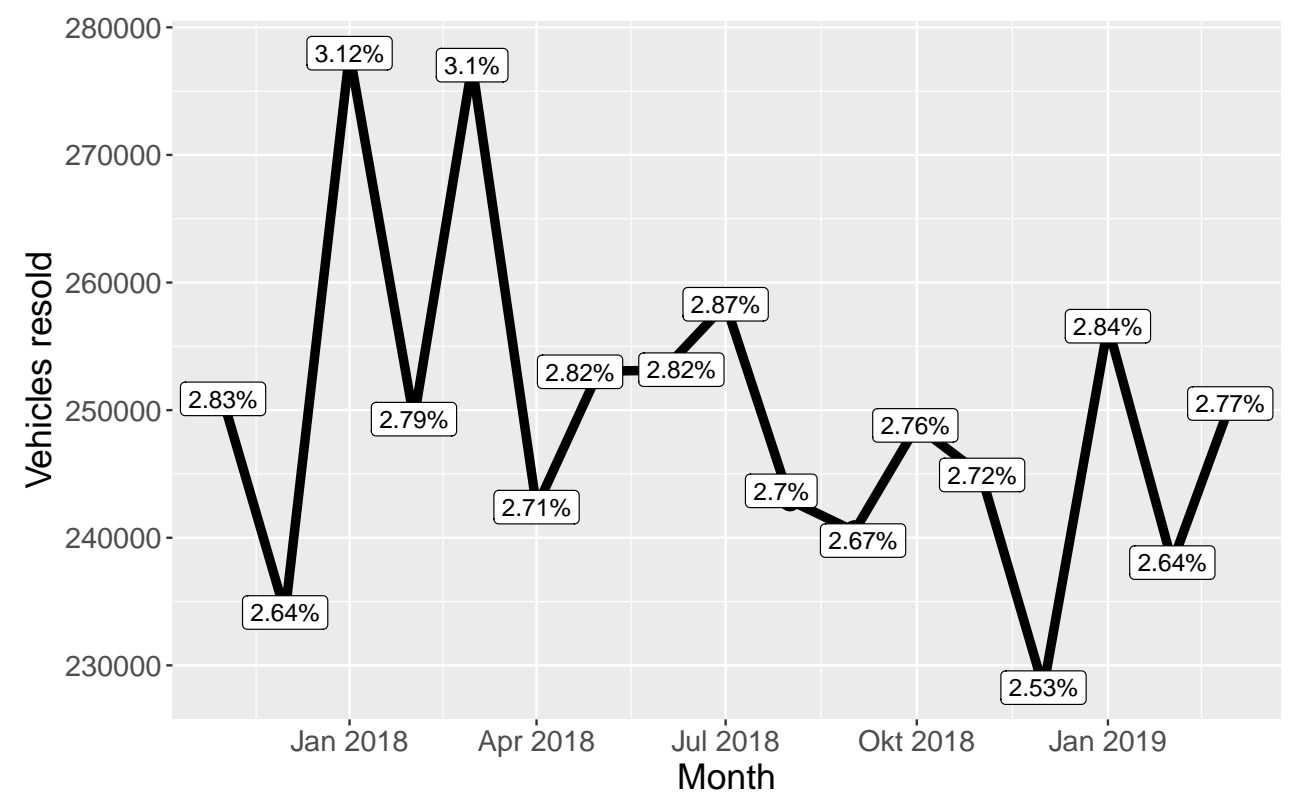

\section{Empirical strategy}

Identification In this section we introduce the econometric methodology used for the main analysis. Any identification strategy of the causal effect of vehicle recalls on vehicle resales must 
take into account why recalls occur in the first place. If recalls were a random event, we could simply compare differences in resales before and after their occurrence. However, vehicle recalls are unlikely to happen randomly; they might be correlated with other (in)visible characteristics and brand-model reputation. For instance, cars from a certain brand might be more likely to be recalled because the manufacturer paid less attention to product quality in general ${ }^{21}$ Similarly, for specific vehicle models, recalls may be correlated with other problems, such as poor driving experience and performance, or higher overall maintenance costs.

Thus, a simple approach that consider changes in resales between different brands or models might also capture broader brand or model specific characteristics that are not linked to the recall, but do affect the resale probability and prices. In an ideal setting, this issue would be resolved by comparing two groups of identical cars, of which only one received a recall. Our identification strategy mimics this approach by exploiting the variation in resales between recalled (treatment) vehicles and non-recalled (control) vehicles belonging to the same version. As defined by EU regulation, and further explained below, vehicles belonging to the same version must be identical in all main vehicle characteristics. As such we are able to mitigate concerns that, within a version, recall occurrence is correlated with the characteristics of the vehicle.

We consider this approach of using the EU vehicle classification system, where vehicle versions are defined in accordance with directives composed and executed by industry experts, preferable to an alternative approach wherein we ourselves would define an 'equivalent vehicle' based on observables. To our knowledge, we are the first to exploit the EU vehicle system classification as part of the identification strategy. This strategy is facilitated by the highly disaggregated and rich nature of the Dutch vehicle registration data. Further information about how vehicle versions are defined in the EU classification system is provided below.

\footnotetext{
${ }^{21}$ There is indeed anecdotal evidence on the correlation between vehicle defects and broader flaws in the production process. A report to the GM board of directors about the vehicle recall due to faulty ignition system underlines general issues within the company: "While GM heard over and over from various quarters [...] that the car's ignition switch led to moving stalls, group after group and committee after committee within GM that reviewed the issue failed to take action or acted too slowly. Although everyone had responsibility to fix the problem, nobody took responsibility. [...] $A$ critical factor in GM personnel's initial delay in fixing the switch was their failure to understand, quite simply, how the car was built." (Valukas, 2014). Recent research has also established a negative effect of firm financial position on recalls of durable goods, including cars (Kini, Shenoy, \& Subramaniam, 2017).
} 
Vehicle version and EU classification Before a specific vehicle can be sold on the European market, vehicle manufacturers are required to obtain an approval. Approvals are classified according to well-defined categories of vehicle type, variant, and version (European Parliament, 2007). Vehicles of the same type are identical in the following aspects: manufacturer, fundamental characteristics of chassis/floor pan, and power plant (e.g., internal combustion/electric/hybrid). Within the same variant, vehicles must also be identical in terms of body style, engine working principle, number and arrangement of cylinders, axles, while only limited variation in engine power and engine size are permitted. Finally, vehicles belonging to the same version are also identical in terms of engine power and size, gear, fuel consumption, seats, and emissions. Thus, within a version, vehicles are close to identical, except for certain optional characteristics, such as color, upgraded interior trim pieces, or additional safety packages.

Though, in principle, two vehicles manufactured many years apart could belong to the same version, this rarely occurs. Rather, due to the rapid technological progress and intense competition in the vehicle sector, the dispersion in vehicle age within the same version is small: we find a mean difference between the first and last-sold vehicle within a particular version of 274 days, and a median difference of 190 days $\sqrt{22}$ As consumers generally understand that different generations of a specific vehicle can be of different quality, and will not consider vehicles that greatly differ in age as 'equivalent vehicles', we consider this an advantageous feature of using the version classification to identify very similar cars.

It is relevant to highlight that the same commercial name is typically used for several vehicle versions, or even variants or types $2^{23}$ Vice versa, manufacturers nearly always advertise all vehicles belonging to the same version under the same commercial name in the market of sale. As the specific version classification is not communicated to the consumer other than through the vehicle certificate, vehicle owners are unlikely aware of this information.

\footnotetext{
${ }^{22}$ Changes in vehicle regulatory requirements contribute to this, as vehicle manufacturers are obliged to terminate new vehicle sales whenever vehicles of that particular version are no longer compatible with the latest regulatory requirements.

${ }^{23}$ For example, the same commercial name "Ford Focus Wagon 1.6 Ecoboost Titanium 150PK" can belong to a vehicle with type approval "e13*2007/46*1138*00", but also to a vehicle with type approval "e13*2007/46*1138*03".
} 
Variation in recalls and sample used Our empirical strategy allows us to identify the effect of receiving information about a recall on the probability of vehicle resale only if there exists sufficient variation in recalls across different vehicles belonging to the same version - i.e., if within same version some vehicles are recalled and some are not. Figure 4, which illustrates the distribution of versions based on their share of recalled cars, shows this is the case. As can be seen from the figure, 78 percent of versions never had a vehicle recalled, while for 12 percent of versions, all vehicles had been subject to a recall. Our identification strategy thus relies on exploiting variation in recalls in the remaining 18,789 versions ( $8.93 \%$ of all versions in the sample), as these contain both recalled and non-recalled vehicles.

There are several potential explanations for why of two seemingly identical vehicles, one may be subject to a recall, and the other not. Vehicles from the same versions might have been produced in different plants, in different production cycles, and for these or other reasons have components from different suppliers. Then any variation in the production process across plants or cycles, or simply a poor batch of supplies, may cause variation in recalls within vehicle versions. ${ }^{24}$ This is similar to what occurs for recalls in the food industry, where typically only specific batches or serial numbers of the same product are withdrawn from the market. Because there exist such variation in the need to recall vehicles within the same version, recalls are issued and registered at the level of specific vehicles.

The main dataset we use in our analysis includes 7,852,875 vehicles and 202,637 versions. This dataset includes only vehicles that have either not been recalled or that have been recalled only once; this ensures we have a clear date of the start of the treatment. We exclude also very old vehicles, typically registered for the first time before 2000, for which our dataset does not specify the type-variant-version classification. Finally, there is a very small number of vehicles that disappear from the sample within our sample period. This could be because the vehicle has been scrapped or because it has been stolen. Because we are not able to distinguish between these

\footnotetext{
${ }^{24}$ Communication with the Dutch regulatory authority confirmed this is a credible hypothesis. Additionally, the fact that the EU Rapid Alert System reports for each recall both the range of dates of fabrication and the Vehicle Identifier Numbers of the recalled vehicles is consistent with this hypothesis.
} 
Figure 4: Share of recalled vehicles within version (all recalls up to April 2019)

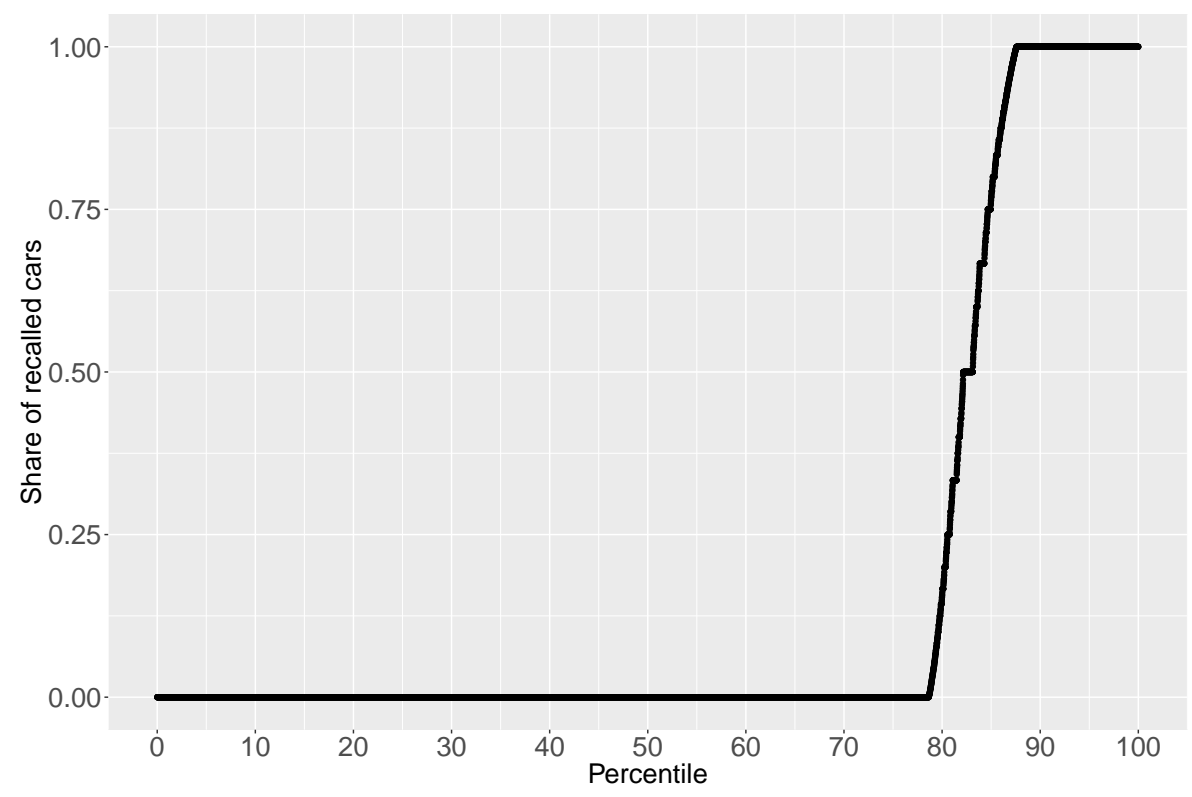

Notes: Plot of the share of recalls within each version, ordered by percentile. Each observation in the graph corresponds to a version. The vertical axis shows the share of vehicles in the version that have been recalled at any time up to early April 2019.

two causes, we drop those vehicles. ${ }^{25}$

Summary statistics and illustrative evidence Table 1 presents several summary statistics for recalled and non-recalled vehicles. Specifically, we compare vehicle characteristics, new vehicle listed price, and age of recalled and non-recalled vehicles belonging to the same versions, by reporting average values of within-version means. ${ }^{26}$ In most cases, there is no statistically significant difference between recalled and non-recalled vehicles. We do however observe a small difference in vehicle age (3 months). This could signal that cars in earlier production cycles are more likely to be recalled.

We present some illustrative evidence regarding the effect of recalls on resales in the form of an event study graph. To construct this graph, we calculate the unweighted average of the share

\footnotetext{
${ }^{25}$ In the Netherlands, most vehicles get exported towards the end of their lifetime rather than scrapped. We retain exported vehicles in our sample until the date of export.

${ }^{26} \mathrm{We}$ do not compare the entire fleet of recalled vehicles to non-recalled vehicles as our identification strategy relies on exploiting variation in recalls within version. Additionally we do not report on vehicle characteristics such as fuel economy, as they are by definition equal within version.
} 


\begin{tabular}{l|cc|c} 
& \multicolumn{2}{|c}{ Average } & T-test \\
List price & Non-recalled & Recalled & \\
Num. seats & 53052.06 & 53510.21 & 0.449 \\
Num. doors & 5.06 & 5.05 & 0.633 \\
Num. cylinders & 4.26 & 4.26 & 0.804 \\
Engine displacement & 1875.24 & 4.23 & 0.205 \\
Mass empty vehicle & 1444.94 & 1890.00 & 0.190 \\
Mass on road & 1544.94 & 1444.64 & 0.944 \\
Max. mass allowed & 2088.43 & 2086.02 & 0.645 \\
Age (months) & 45.70 & 48.26 & 0.000
\end{tabular}

Note: Test of equality of means for averages of recalled and not recalled vehicles within the same version. The values reported are the averages of the mean values for recalled and not recalled vehicles within each version, and the p-value of the T-test. Only versions with both recalled and not recalled vehicles are considered.

Table 1: Summary statistics by vehicle version, recalled and not recalled vehicles

of resales within a version for each month, separately for cars that have been recalled and cars that have not. We assign a placebo recall for non-recalled cars using the modal date of recall for recalled vehicles belonging to the same version.

The resulting event study graph is presented in Figure 5. On the horizontal axis, the zero marks the month in which the recall has been issued. Before the recall is issued, we observe very similar dynamics for recalled and non-recalled vehicles. After the recall however, we see a large drop in resales for recalled cars. Overall, the event study graph offers compelling evidence of the presence of an effect of recalls on vehicle resales.

Econometric specification We begin our analysis with a baseline difference in differences specification to estimate the effect of vehicle recalls on vehicle resales:

$$
\text { Resold }_{i j t}=\alpha+\beta \text { RecalledPost }_{i t}+\gamma \text { Recalled }_{i}+\delta \text { Age }_{i t}+\zeta \text { DSale }_{i t}+\eta_{t}+\theta_{j}+u_{i j t} .
$$

The dependent variable Resold $d_{i j t}$ is equal to 1 if vehicle $i$ changed ownership registration in year-month $t$, and equal to zero otherwise. Each vehicle belongs to version $j$. Our treatment 
Figure 5: Effect of vehicle recalls on resales: recalled and non-recalled vehicles

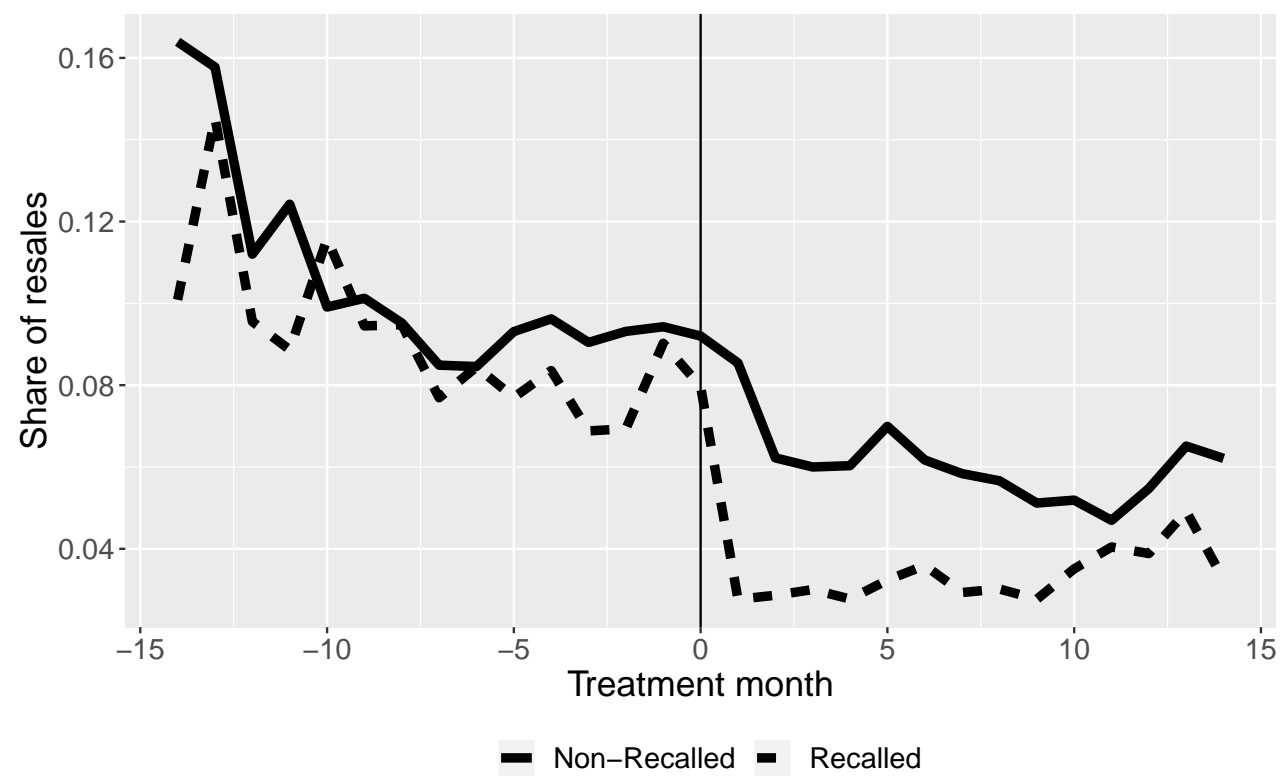

Notes: Difference in differences event study graph. The vertical axis displays the unweighted average resale share by version. The horizontal axis displays the distance in months from the occurrence of the recall. Data includes only versions with both recalled and non-recalled vehicles. Placebo recall starting date for non-recalled vehicles is based on the modal recall date of the recalled vehicles within the same version.

indicator, RecalledPost ${ }_{i t}$, has value 1 if a car has been recalled at time $t$ or before, and 0 otherwise. Variable Recalled $_{i}$ is an indicator for the car having been recalled at any point in time up to March 2019, which is the last date of observation in our dataset. This indicator controls for any compositional differences between ever recalled and never recalled vehicles. We also control for the age of the car in years, $A g e_{i t}$, and the time in years since the last registration change, DSale $i t$. Additionally, we include year-month fixed effects $\eta_{t}$ and version fixed effects $\theta_{j}$.

We estimate (5) using a linear probability model. Thus, our coefficient of interest $\beta$ measures the effect of a recall on the probability of a vehicle to be sold in a given month.

Our identification strategy relies on recalled vehicles of a given vehicle version to be "virtually identical' to non-recalled vehicles belonging to the same version. Table 1 shows that there are no difference between recalled and non-recalled vehicles within a version in terms of new vehicle list price and other observable physical characteristics. Yet, one might be concerned that certain characteristics unobservable to us, but observable to the consumer (such as options), are systematically 
different between those two groups, and correlated with recall and resale incidence. To address this concern we include an interaction between the version fixed effects and the indicator on whether the car has been recalled or not at any point in time before the end of March 2019. We refer to this set of fixed effects in the paper as version-recall fixed effects.

Trends in the secondary vehicle market may vary across market segments. In addition, a large recall, such as the Takata airbag recall, might worsen the overall reputation and desirability of a particular brand or a model, regardless of whether a specific vehicle has been actually recalled or not. Any of these spillover effects would be captured by our coefficient of interest if they are correlated with the timing of a recall. To account for this, and improve the robustness of our identification strategy, we add a set of version-time fixed effects to the baseline specification $5^{27}$

Following the discussion above, we estimate the four different specifications. These are (i) the baseline specification (5), which includes only version and time fixed effects; (ii) a specification with version-recall and time fixed effects; (iii) a specification with version-time fixed effects; (iv) a specification with both version-recall and version time-fixed effects. Specification (iv) is our preferred specification and can be represented as follows:

$$
\text { Resold }_{i j r t}=\alpha+\beta \text { RecalledPost }_{i t}+\delta \text { Age }_{i t}+\zeta \text { DSale }_{i t}+\phi_{j r}+\psi_{j t}+u_{i j r t},
$$

where the subscript $r$ represents whether the car is ever recalled or not, $\phi_{j r}$ are version-recall fixed effects and $\psi_{j t}$ are version-time fixed effects.

The different specifications exploit different sources of variation in recalls in the data. In particular, for the estimation of $\beta$, our preferred specification (iv) relies only on vehicle versions with a recall occurring during our sample period from November 2017 to March 2019, and for which only a subset of cars has been recalled ${ }^{28}$ Hence, our identifying assumption for specification (iv)

\footnotetext{
${ }^{27}$ Such version-time fixed effects will also account for any policy changes that may impact some versions more than others, and capture any effects of the recall on overall brand reputation.

${ }^{28}$ Conversely, our baseline specification (5) relies on the resale variation within a broader set of versions. As for our preferred specification, this includes any variation in recalls within version during our sample period. It additionally includes variation within version in recalls occurred before our sample period, as well as variation over time in any recalls occurred during our sample period.
} 
is that in the counterfactual scenario of no recalls, within a vehicle version, the evolution of resales over time would have been the same for recalled and non-recalled vehicles. To provide evidence for the validity of this assumption, we perform a parallel trend test for all four specifications. For specification (iv), this parallel trend test requires us to estimate the following:

$$
\operatorname{Resold}_{i j r t}=\alpha+\sum_{d} \beta_{d} \text { Recalled }_{i} * \operatorname{TtR}_{d}+\delta \text { Age }_{i t}+\zeta \text { DSale }_{i t}+\phi_{j r}+\psi_{j t}+u_{i j r t},
$$

where $T t R_{d}$ represents the time distance (per three months) from the occurrence of the recall. Here, $d=-4,-3, \ldots+3$, with 0 being the first three months after the recall and -1 being the omitted category ${ }^{29}$ Then, whenever the estimated $T t R_{d}$ is significantly different from zero for $d<0$, the parallel trend assumption is violated.

\section{Results}

Parallel trend test The results of the parallel trend tests are presented in Figure 6. It shows that, prior to the recall, there is no statistically significant difference in resale rates between vehicles that are recalled and vehicles that remain non-recalled (95\% confidence level) ${ }^{30}$ In our preferred specification (iv) with version-recall and version-time fixed effects, we find no evidence of pretrends.

Following the recall, we observe a significant decline in resales in the second quarter postrecall, followed by no effect or an increase afterwards. For specifications (iii) and (iv) the magnitude of this effects is larger, while qualitatively the main conclusions are unchanged.

An interpretation of these results is that the recall leads to a delay in resales. A potential explanation for this is that owners prefer to repair the vehicle prior to offering it for sale. Illustrative

\footnotetext{
${ }^{29}$ To ensure enough variation in the year-month of observation of the vehicles within each category, we group in a unique category all vehicles observed more than 9 months before the recall, and we do the same for vehicles observed more than 9 months after the recall.

${ }^{30}$ Under specification (iii) with version-time fixed effects $T t R_{-3}$ is significantly different from zero (90\% confidence level) six to nine months prior to the recall (see bottom left quadrant). The remaining pre-trend coefficients are not significant at the $10 \%$ level
} 
evidence regarding resale trends for repaired and unrepaired vehicles however fails to lend support to this hypothesis (see Appendix C for an analysis).

Figure 6: Parallel trend test
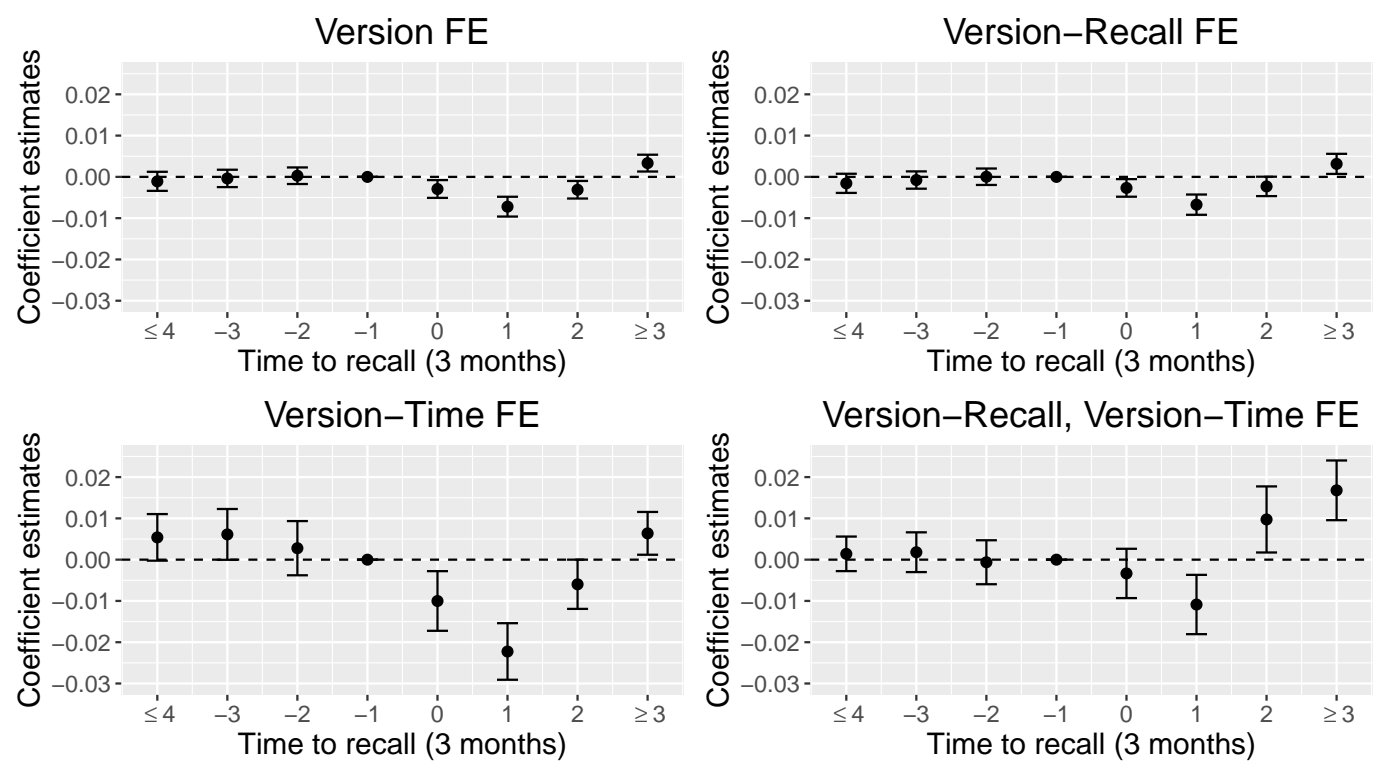

Notes: Results from the parallel trend test (equation (7)). The horizontal axis shows the time (in units of three months) since the start of the recall (time 0 ). Each coefficient represents the change in probability of a resale occurring during each three month time period. Time period -1 is the omitted coefficient. The four panels show the results from using different groups of fixed effects. Standard errors at the vehicle type-time level. The bars represent $95 \%$ confidence intervals.

Overall results Our estimation results for the overall effect of a recall on resales are presented in Table 2. We present the results for specifications (i) through (iv), starting from the baseline model (i) described in equation (5) up to our preferred model (iv) as given by equation (6). The coefficient RecalledPost captures the change in the monthly resale probability of treatment (recalled) vehicles, compared to control (not recalled) vehicles, following the recall. To provide a comparison, the average monthly resale probability for control vehicles is $3.31 \%$. All estimations show an overall decline in resales following the recall. However, the coefficient of interest is not statistically significant for specifications (iii) and our preferred specification (iv).

This lack of a significant effect need not imply that market participants ignore the recall, or that the recall does not provide additional information on vehicle quality; the theoretical framework 


\begin{tabular}{l|cccc} 
& (i) & (ii) & (iii) & (iv) \\
\hline RecalledPost & $-0.0022^{* * *}$ & $-0.0030^{* * *}$ & -0.0006 & -0.0013 \\
& $(0.0007)$ & $(0.0009)$ & $(0.0012)$ & $(0.0023)$ \\
Recalled & $-0.0047^{* * *}$ & & $-0.0062^{* * *}$ & \\
& $(0.0007)$ & & $(0.0011)$ & \\
\hline & \multicolumn{5}{|}{} \\
\hline Version-Recall FE & No & Yes & No & Yes \\
Version-Time FE & No & No & Yes & Yes
\end{tabular}

Results from specifications (i)-(iv) with a linear probability model. The dependent variable is an indicator for whether the car has been resold in a given period. Coefficient RecalledPost identifies the change in monthly resale probability due to a recall. Coefficient Recalled controls for time-invariant compositional differences in resales between recalled and non-recalled cars. The total sample size is $126,698,700$ observations for $7,852,875$ unique vehicles. Standard errors are clustered at the vehicle type-time level.

Table 2: Overall effect of recall

established that the overall effect of recalls on resales can go either way. In particular, our model highlights that recalls can improve sorting and thus increase vehicle transactions. Simultaneously however, a recall might change the buyer's expectation of the asymmetrically observed vehicle quality, which can reduce transactions.

As argued in Section 3, considering the vehicle market as a whole may disguise important heterogeneities across vehicle market segments. Below, we consider such heterogeneities. In particular, we analyse whether the effect of a recall differs across groups of vehicles of different quality. Because there exists no single objective measure of vehicle quality, we instead consider three different proxies for vehicle quality. These are (i) new vehicle listed price, (ii) history of defects identified during mandatory inspections, and (iii) a brand reliability score. These proxies all capture a slightly different dimension of vehicle quality. For instance, the vehicle listing price can be considered an indicator of the expected quality of a new model or version, while the inspection results may more accurately reflect the recent conditions of the specific vehicle 31

\footnotetext{
${ }^{31}$ We additionally considered heterogeneity by recall characteristics, in particular the vehicle part involved in the recall, number of vehicles involved in each recall episode, and risk type as communicated by RDW. We did not find clear evidence of systematic heterogeneity in those dimensions.
} 
Results by new vehicle list price The vehicle price is the first obvious candidate for a proxy for vehicle quality: previous studies have shown a positive correlation between list price and the various dimensions of vehicle quality such as comfort, efficiency, safety and reliability (McCarthy, 1996). As such, list price can be considered a broad measure for vehicle quality at the beginning of the vehicle's lifetime. Information about the list price is readily available to buyers through the RDW database, even for older cars.

We split the sample of used vehicles into two parts according to the vehicle's list price when purchased new. We adopt a threshold value of 30,000 EUR ${ }^{32}$ and estimate the effect of a recall on resales separately for each of the two vehicle price categories. Differences in price within version are limited; all vehicles belonging to the same version typically also belong to the same price group ${ }^{33}$ The identification strategy and regression specification are the same as in the main results in Table $2^{34}$

Table 3 presents the results by price category. Panel A shows the effect of a recall for cheaper vehicles, while Panel B shows the effect for more expensive vehicles. Under our preferred specification, less expensive vehicles experience an overall increase in resale probability of 1.08 percentage points following a recall, while expensive vehicles see a decline in resale probability of 1.32 percentage points.

Effect of recalls by inspection defects While the list price can be considered to broadly reflect the quality of the vehicle when new, it provides little indication of the evolution of quality over time, nor signals the 'lemons' within the vehicle pool. Poor maintenance routines or intensive vehicle use can, for instance, affect the quality of car. Similarly, whether a vehicle belongs to a 'poor batch' is only revealed over time. To obtain a measure of vehicle-specific quality after the first registration, we consider the results from the Dutch mandatory vehicle inspections.

\footnotetext{
${ }^{32}$ This threshold value corresponds approximately to the median real purchase price of our sample. To ensure comparability, we correct all prices for inflation, using 2016 as a base year.

${ }^{33}$ Whenever, within the same version, we observe cars with prices both above and below the 30,000 EUR threshold, we treat them as if they belonged to a different version.

${ }^{34}$ For both market segments, we observe sufficient variation in recall rates within version: below the 30,000 EUR threshold, about 3995 (9.52\% of the versions in the segment) versions have both and recalled and non-recalled vehicles; for cars above the threshold this applies to 12,262 versions (11.08\%) (based on March 2019 vehicle fleet).
} 


\begin{tabular}{|c|c|c|c|c|}
\hline & \multicolumn{4}{|c|}{ Panel A: Price $<30 \mathrm{~K}$ EUR } \\
\hline & (i) & (ii) & (iii) & (iv) \\
\hline \multirow[t]{2}{*}{ RecalledPost } & $0.0043^{* * *}$ & $0.0046^{* * *}$ & $0.0034^{*}$ & $0.0108^{* * *}$ \\
\hline & $(0.0008)$ & $(0.0009)$ & $(0.0018)$ & $(0.0041)$ \\
\hline \multirow[t]{4}{*}{ Recalled } & $-0.0086^{* * *}$ & & $-0.0080^{* * *}$ & \\
\hline & $(0.0009)$ & & $(0.0018)$ & \\
\hline & \multicolumn{4}{|c|}{ Panel B: Price $\geq 30 \mathrm{~K}$ EUR } \\
\hline & (i) & (ii) & (iii) & (iv) \\
\hline \multirow[t]{2}{*}{ RecalledPost } & $-0.0078^{* * *}$ & $-0.0096^{* * *}$ & $-0.0056^{* *}$ & $-0.0132^{* * *}$ \\
\hline & $(0.0015)$ & $(0.0016)$ & $(0.0023)$ & $(0.0031)$ \\
\hline \multirow[t]{2}{*}{ Recalled } & $-0.0041^{* * *}$ & & $-0.0056^{* *}$ & \\
\hline & $(0.0015)$ & & $(0.0023)$ & \\
\hline Version-Recall FE & No & Yes & No & Yes \\
\hline Version-Time FE & No & No & Yes & Yes \\
\hline
\end{tabular}

Results from specifications (i)-(iv) with a linear probability model, for two separate subsamples of vehicles with a real listed price of below 30K EUR and vehicles with a real listed price of 30K EUR or more. The dependent variable is an indicator for whether the car has been resold in a given period. Coefficient RecalledPost identifies the change in monthly resale probability due to a recall. Coefficient Recalled controls for timeinvariant compositional differences in resales between recalled and non-recalled cars. For Panel A the total sample size is of 51,637,432 observations for 3,227,084 unique vehicles. For Panel B the total sample size is of $28,005,073$ observations for $1,835,556$ unique vehicles. Standard errors are clustered at the vehicle type-time level.

Table 3: Results by real vehicle listing price, separate regressions

Like in other European countries, in the Netherlands, vehicles are subject to a regular mandatory inspection. This inspection, referred to as the APK, often reveals defects that must be fixed before the car is allowed back on the road. APK inspectors are required to communicate results to the RDW. In an effort to improve transparency on the market, the RDW publishes these results through its standard channels. As such, the APK results constitute a freely available vehiclespecific signal of current vehicle quality ${ }^{35}$ In the remainder, we will consider vehicle with a recent history with defects as one with a lower vehicle quality.

The first mandatory APK test is due 4 years after the first vehicle registration, and is subsequently repeated every 1-2 years depending on the age of the vehicle. To ensure that all cars

\footnotetext{
${ }^{35}$ Any defects that are identified during other inspections, or defects insufficiently severe to cause noncompliance with regulations are not registered by the RDW. Hence, the absence of defects during the APK need not imply the car had not defects whatsoever.
} 
considered had at least one inspection, we restrict the sample to vehicles of 4 years or older. We then run the analysis separately for two groups: cars that had no defects during their last inspection, and cars that had one or more defects $\sqrt[36]{36}$ The results are presented in Table 4

Results from Table 4 are in line with those obtained in the analysis using listed price: higher quality vehicles (no APK defects) experience a reduction in resale probability as a consequence of the recall, whereas resales for lower quality vehicles (one or more defects found during the inspection) increase.

\begin{tabular}{l|cccc} 
& \multicolumn{4}{|c}{ Panel A: No defects } \\
& (i) & (ii) & (iii) & (iv) \\
\hline RecalledPost & $-0.0010^{* *}$ & $-0.0012^{* *}$ & $-0.0041^{* * *}$ & $-0.0140^{* * *}$ \\
Recalled & $(0.0004)$ & $(0.0005)$ & $(0.0008)$ & $(0.0021)$ \\
& $-0.0075^{* * *}$ & & $-0.0046^{* * *}$ & $(0.0007)$ \\
& $(0.0004)$ & & \\
& \multicolumn{5}{|c}{ Panel B: One or more defects } \\
& $(1)$ & $(2)$ & $(3)$ & $(4)$ \\
\hline RecalledPost & $0.0015^{* * *}$ & $0.0017^{* * *}$ & $0.0014^{* * *}$ & $0.0016^{* *}$ \\
& $(0.0002)$ & $(0.0003)$ & $(0.0005)$ & $(0.0008)$ \\
Recalled & $-0.0005^{*}$ & \multicolumn{3}{c}{$-0.0004^{* * *}$} \\
& $(0.0003)$ & & $(0.0004)$ & \\
& \multicolumn{5}{c}{ No } & Yes \\
\hline Version-Recall FE & No & Yes & Yes \\
Version-Time FE & No & No & Yes
\end{tabular}

Results from specifications (i)-(iv) with a linear probability model, for two separate subsamples of vehicles with no defects found during the last APK inspection (Panel A) and with one or more defects found (Panel B). The dependent variable is an indicator for whether the car has been resold in a given period. Coefficient RecalledPost identifies the change in monthly resale probability following a recall. Coefficient Recalled controls for time-invariant compositional differences in resales between recalled and non-recalled cars. For Panel A the total sample size is of 28,245,023 observations for $2,302,428$ unique vehicles. For Panel B the total sample size is of $70,107,490$ observations for 4,522,001 unique vehicles. Standard errors are clustered at the vehicle type-time level.

Table 4: Results by brand rating, separate regressions

\footnotetext{
${ }^{36}$ About $28.72 \%$ of the observations considered did not have defects in the previous inspection. About $39.71 \%$ had one defect, $18.29 \%$ had two defects and the remaining $13.29 \%$ had three or more. The average number of defects is 1.253. We maintain sufficient variation in recall rates within version: for cars with no defects, about 7452 (7.40\% of the versions in the segment) versions have both and recalled and non-recalled vehicles; for cars with one or more defects this applies to 7168 versions (5.40\%) (based on March 2019 vehicle fleet).
} 
Effect of recalls by reliability score The final measure of vehicle quality we consider is the 2017 manufacturer reliability score from the Consumentenbond, the main Dutch consumer association. The Consumentenbond surveys Dutch vehicle owners on whether their vehicle had a defect in the previous year. If so, it then asks the respondent to specify which part was affected, and whether the defect prevented them from driving, allowed them to drive but required immediate maintenance, or maintenance could be postponed. Based on the survey results, the Consumentenbond construct a reliability score from 5 to 9 for the 24 main vehicle brands ${ }^{37}{ }^{38}$ The manufacturer reliability score can be considered a measure of the amount of maintenance the car would likely need in the future, and as such a measure of vehicle quality. There exists substantial heterogeneity in the reliability of cars across manufacturers and vehicle makes. To capture this heterogeneity, we split our sample in two groups: cars with a low brand reliability rating (below 7), and cars with a high rating (7 or higher) ${ }^{39}$

Under our preferred specification with version-recall and version-time fixed effects, the results in Table 5 show evidence of a decrease in resale rates following a recall for cars of brands with a high reliability rating. This result is consistent with the previous results considering alternative dimensions of quality. For cars of brands with low reliability rating, the coefficient is positive, but not significant, and does not seem robust across the different specifications.

The analysis using brand reliability thus offers less robust evidence in favor of heterogeneous responses to recalls than the analysis using real listed prices or vehicle inspection defects. A possible explanation for this is that the reliability ratings by Consumentenbond are less salient to both buyers and sellers, as they are not included in the report produced on the government or private licence plate search engines. Obtaining the brand rating instead would require the consumer

\footnotetext{
${ }^{37}$ The survey explicitly asks respondents not to include maintenance due to vehicle accidents, but does not mention recalls. It is thus possible that some of the responses in the questionnaire concerned maintenance due to recalls. Yet, recalls never require immediate repairs, which limits their weight in the brand reliability score. As we use results from a survey prior to our sample period, recalls in our sample period do not affect the survey results.

${ }^{38}$ Recent scores can be found at www.consumentenbond.nl/test/auto-fiets-reizen/automankementen We use the scores from 2017, and thank the Consumentenbond for sharing this data with us.

${ }^{39}$ As for the previous proxies of quality, we observe sufficient variation in recalls within version for each segment. On March 2019, for the segment with brand reliability score below 7, we observe 9483 versions $(8.73 \%$ of the versions in the segment) with both recalled and non-recalled cars. For the segment with brand reliability score equal or above 7 we observe 6806 versions (8.84\%) (based on March 2019 vehicle fleet).
} 
to check either the Consumentenbond report directly, or a news article reporting on it.

All in all, our results indicate substantial heterogeneities in the implications of vehicle recalls across market segments: following a recall, resales of high-quality vehicles fall, while resales of low-quality vehicles (weakly) increase. In light of our theoretical framework, this suggests that recalls primarily lead to increased sorting in the lower-end of the vehicle market. Instead, for the high-quality vehicles, exacerbation of the information asymmetries seems to dominate the sorting effect, explaining why the resale probability decreases.

In our interpretation of the analysis' results we maintain the conventional assumption that recalls constitute a negative signal about quality of the recalled vehicle. Yet, one could argue that, if managed well, recalls might improve the quality perception of either the specific vehicle and/or the overall brand. As our empirical strategy controls for version-specific time trends, any positive or negative change in brand reputation does not affect our results. Even though we are not aware of literature evidence that establishes that a recall is regularly considered a positive signal of the quality of a specific recalled car, we cannot rule out this possibility. Further research would be required to assess whether such 'positive recalls' could serve as an alternative explanation for the observed negative effect of recalls on resales in the higher-quality market segments.

Effect on depreciation factor So far our analysis has focused on the effect on the recall on the probability that a vehicle will be resold. Beyond the resale likelihood, recalls may also affect prices on the used car market. Here again the theoretical framework signals that the direction of this effect is not immediate. More specifically, while the recall unambiguously decreases resale prices through the adverse selection channel, it has an ambiguous effect on the used vehicle price through the sorting channel $4^{40}$ Hence, the net effect of recalls on used vehicle prices is theoretically ambiguous, and remains an empirical question.

Our dataset does not include information on vehicle transaction prices. Instead, to assess the effect of recalls on prices, we gathered data on used vehicle asking prices from one of the largest

\footnotetext{
${ }^{40}$ This is further explained in Appendix $B$, where we show that, conditional on a vehicle transaction taking place, the effect of a recall on resale prices depends on parameter values.
} 


\begin{tabular}{|c|c|c|c|c|}
\hline & \multicolumn{4}{|c|}{ Panel A: Brand rating below 7} \\
\hline & (i) & (ii) & (iii) & (iv) \\
\hline \multirow[t]{2}{*}{ RecalledPost } & -0.0017 & $-0.0026^{*}$ & 0.0007 & 0.0055 \\
\hline & $(0.0011)$ & $(0.0015)$ & $(0.0014)$ & $(0.0035)$ \\
\hline \multirow[t]{4}{*}{ Recalled } & $-0.0035^{* * *}$ & & $-0.0062^{* * *}$ & \\
\hline & $(0.0011)$ & & $(0.0014)$ & \\
\hline & \multicolumn{4}{|c|}{ Panel B: Brand rating equal or above 7} \\
\hline & (i) & (ii) & (iii) & (iv) \\
\hline \multirow[t]{2}{*}{ RecalledPost } & $-0.0017^{*}$ & $-0.0028^{* * *}$ & -0.0001 & $-0.0082^{* * *}$ \\
\hline & $(0.0009)$ & $(0.0010)$ & $(0.0021)$ & $(0.0025)$ \\
\hline \multirow[t]{2}{*}{ Recalled } & $-0.0072^{* * *}$ & & $-0.0083^{* * *}$ & \\
\hline & $(0.0010)$ & & $(0.0021)$ & \\
\hline Version-Recall FE & No & Yes & No & Yes \\
\hline Version-Time FE & No & No & Yes & Yes \\
\hline
\end{tabular}

Results from specifications (i)-(iv) with a linear probability model, using three separate subsamples of vehicles with a brand reliability rating below 7 (Panel A), and a rating from 7 and higher (Panel B). The dependent variable is an indicator on whether the car has been resold in a given period. Coefficient RecalledPost identifies the change in monthly resale probability due to a recall. Coefficient Recalled controls for timeinvariant compositional differences in resales between recalled and non-recalled cars. For Panel A the sample size is of 68,396,306 observations for 4,234,740 unique vehicles. For Panel B the sample size is of 51,541,966 observations for 3,192,213 unique vehicles. Standard errors are clustered at the vehicle type-time level.

Table 5: Results by brand rating, separate regressions

online marketplace active in the Netherlands, from May 2018 through the end of our sample period of March 2019. We consider all vehicles with a valid license plate, which allows us to match vehicles to the registration database and identify duplicate resales within short periods of time ${ }^{41}$ In total, we obtained a sample of 394,661 vehicles, which amounts to an average of 35,878 vehicles offered on the market each month. Comparing this to the vehicle registration database, this corresponds to about $15 \%$ of the total number of transactions in the period considered.

Used vehicle prices are closely linked to the original value of the vehicle. Hence, rather than estimating the effect of recalls on the vehicle asking price, we estimate the effect on the vehicle depreciation factor. This factor is calculated as the ratio between the asking price and the vehicle

\footnotetext{
${ }^{41}$ Vehicles without a valid licence plate are typically new vehicles, sold directly by the dealer. We also remove very old vehicles with no information about the version they belong to or the listed price.
} 
list price.$^{42}$ where both prices are expressed in real terms. We adopt the same empirical strategy as for the analysis of the resale probability. Even though our data is no longer in a panel format, positive supply of both recalled and non-recalled vehicles belonging to the same version allows us to use the same identification strategy. ${ }^{43}$ Thus, the corresponding equation for our baseline model (5) is the following:

$$
\begin{aligned}
\text { Dprate }_{i j z t}= & \alpha+\beta \text { RecalledPost }_{i t}+\gamma \text { Recalled }_{i}+\delta \text { Age }_{i t}+\zeta \text { Dealer }_{i t}+ \\
& \nu_{1} k m_{i t}+\nu_{2} k m_{i t}^{2}+\xi_{z}+\eta_{t}+\theta_{j}+u_{i j t}
\end{aligned}
$$

where Dprate $_{i j z t}$ is the depreciation rate of vehicle $i$ with version $j$, offered for sale in zip code $z$ at year-month $t$. The main difference to our previous specification (5) are the inclusion of (i) a dummy Dealer $i$, which captures whether the seller is a car dealership or not, (ii) a linear and quadratic term for total $\mathrm{km}$ driven by the listed vehicle, $k m_{i t}$ and $k m_{i t}^{2}$, and (iii) zip code fixed effects $\xi_{z}$, to capture any regional differences in used vehicle markets. ${ }^{44}$ The time since the last sale is not included as arguably it does affect on the asking price.

Similarly, the equivalent of our preferred specification (6) with version-recall and version-time fixed effects is the following:

$$
\begin{aligned}
\text { Drate }_{i j r z t}= & \alpha+\beta \text { RecalledPost }_{i t}+\delta \text { Age }_{i t}+\zeta \text { Dealer }_{i t}+ \\
& \nu_{1} k m_{i t}+\nu_{2} k m_{i t}^{2}+\xi_{z}+\phi_{j r}+\psi_{j t}+u_{i j r t} .
\end{aligned}
$$

Results for all four specifications (baseline model, version-recall, version-time, version-recall and version-time) are shown in Table 6, Our results show small and generally insignificant effects of recalls on vehicle depreciation rates. In Appendix $\mathrm{D}$ we additionally perform separate regressions by market segment, analogous to tables 3, 4, and 5. We do not find evidence of systematic

\footnotetext{
${ }^{42}$ The list price is the price for which the vehicle was listed when it was purchased new. This list price may slightly deviate from the price originally paid for the vehicle due to e.g., dealer discounts.

${ }^{43}$ Our sample includes 434,948 control vehicles that never received a recall, and 17,998 and 65,333 vehicles that were entered in circulation before and after receiving a recall, respectively.

${ }^{44}$ We include a total of 3815 zip code fixed effects.
} 
heterogeneity in the price response across market segments.

As a final note, while we do not find evidence that recalls affect used vehicle depreciation factors, we cannot rule out that transaction prices are unaffected, as asking prices typically deviate from transaction prices e.g., due to haggling, which may be affected by the recall history.

\begin{tabular}{l|cccc} 
& (i) & (ii) & (iii) & (iv) \\
\hline RecalledPost & -0.0005 & 0.0024 & $-0.0050^{*}$ & 0.0031 \\
& $(0.0015)$ & $(0.0016)$ & $(0.0030)$ & $(0.0035)$ \\
Recalled & 0.0019 & & $0.0056^{*}$ & \\
& $(0.0022)$ & & $(0.0031)$ & \\
\hline Version-Recall FE & No & Yes & No & Yes \\
Version-Time FE & No & No & Yes & Yes
\end{tabular}

Effect of the recall on vehicle depreciation factor (ratio of asking price and listed price). Coefficient RecalledPost identifies the change in depreciation factor due to a recall. Coefficient Recalled controls for time-invariant compositional differences in depreciation factor between recalled and non-recalled cars. Standard error clustered at the vehicle type-time level. The sample size is of 518,279 observations of unique vehicles.

Table 6: Effect of recalls on depreciation rate

\section{Concluding comments}

Over the past years, several large vehicle recall episodes have made newspaper headlines. Yet, despite this substantial attention to recalls by the media and general public, the effect of recalls on vehicle markets remains understudied. In this paper, we shed light on this topic by considering the effect of vehicle recalls in the context of the Dutch secondary vehicle market, focusing on the effect of resale rates.

At first glance, our results do not seem to justify the substantial media attention, and consumer discontent, vehicle recalls evoke: for our preferred specification, we find no significant effect of recalls on resale rates and vehicle depreciation rates. These overall effects however disguise substantial heterogeneities, both in time and across market segments. For the first 6 months following a recall, resale rates tend to decline; depending on model specification, they remain constant or increase afterwards. Higher-end vehicles are more likely to experience a decrease in resale rates 
following a recall, while we find evidence for an increase in resale rates for the cheaper and lowerquality market segments.

We argue that recalls constitute an example of new information arrival in the used vehicle market. This information may increase resales through increased sorting, or reduce resales by exacerbating pre-existing adverse selection problems. As such, the reduction in resales identified for high quality vehicle may signal that information arrival may reduce market efficiency in this market segment.

Past research on vehicle recalls has generally assessed the effect of a single, high-profile, recall episode (Ater and Yosef, 2018; Strittmatter and Lechner, 2020). Our analysis, however, establishes substantial heterogeneity in the effect of recalls across time and market segments. Thus, our results suggest that one should exercise caution with making general claims based on single-event studies, as insights may not generalize across vehicle segments and recall episodes.

\section{References}

Ater, I. \& Yosef, N. (2018). The impact of product recalls on the secondary market: Evidence from dieselgate. CEPR Discussion Paper, DP12899.

Bachmann, R., Ehrlich, G., Fan, Y., \& Ruzic, D. (2019). Firms and collective reputation: A study of the volkswagen emissions scandal. NBER Working Paper, 26117.

Barrage, L., Chyn, E., \& Hastings, J. (2020). Advertising and environmental stewardship: Evidence from the bp oil spill. American Economic Journal: Economic Policy, 12(1), 33-61.

Beattie, G., Durante, R., Knight, B., \& Sen, A. (2020). Advertising spending and media bias: Evidence from news coverage of car safety recalls. Management Science.

CBS. (2019). CBS statline. Retrieved from https://opendata.cbs.nl/

Cox Automotive. (2018). 2018 used car market report. 
Daily Mail. (2017, April 12). More than a million dangerous cars are still on britain's roads because essential repairs following recalls have not been carried out. Retrieved from https://www. dailymail.co.uk/news/article-4403458/More-million-dangerous-cars-British-roads.html

Eichholtz, P., Kok, N., \& Quigley, J. M. (2013). The economics of green building. Review of Economics and Statistics, 95(1), 50-63.

Elfenbein, D. W., Fisman, R., \& McManus, B. (2015). Market structure, reputation, and the value of quality certification. American Economic Journal: Microeconomics, 7(4), 83-108.

European Commission. (2020). Safety gate: Rapid alert system for dangerous nonfood products. Retrieved from https://ec.europa.eu/consumers/consumers_safety/safety_products/rapex/ alerts

European Parliament. (2001). Directive 2001/95/EC.

European Parliament. (2007). Directive 2007/46/EC.

Ferrer, R. \& Perrone, H. (2017). Consumers' costly responses to product-harm crises. Working Paper.

Freedman, S., Kearney, M., \& Lederman, M. (2012). Product recalls, imperfect information, and spillover effects: Lessons from the consumer response to the 2007 toy recalls. Review of Economics and Statistics, 94(2), 499-516.

Hammond, R. G. (2013). Sudden unintended used-price deceleration? the 2009-2010 toyota recalls. Journal of Economics \& Management Strategy, 22(1), 78-100.

Hartman, R. S. (1987). Product quality and market efficiency: The effect of product recalls on resale prices and firm valuation. The Review of Economics and Statistics, 69(2), 367.

Hendel, I. \& Lizzeri, A. (1999). Adverse selection in durable goods markets. American Economic Review, 89(5), 23.

Houde, S. (2018). How consumers respond to product certification and the value of energy information. The RAND Journal of Economics, 49(2), 453-477.

iSeeCars. (2018). 'car recalls: How to check for recalls by VIN. Retrieved from https://www. iseecars.com/car-recall-study 
Jin, G. Z. \& Leslie, P. (2003). The effect of information on product quality: Evidence from restaurant hygiene grade cards. The Quarterly Journal of Economics, 118(2), 409-451.

Kini, O., Shenoy, J., \& Subramaniam, V. (2017). Impact of financial leverage on the incidence and severity of product failures: Evidence from product recalls. The Review of Financial Studies, 30(5), 1790-1829.

Lewis, G. (2011). Asymmetric information, adverse selection and online disclosure: The case of ebay motors. American Economic Review, 101(4), 1535-46.

Liu, Y. \& Shankar, V. (2015). The dynamic impact of product-harm crises on brand preference and advertising effectiveness: An empirical analysis of the automobile industry. Management Science, 61(10), 2514-2535.

McCarthy, P. S. (1996). Market price and income elasticities of new vehicle demands. The Review of Economics and Statistics, 78(3), 543

NHTSA. (2017). Report to congress: Vehicle safety recall completion rates report.

NHTSA. (2019). 2019 recall annual report. Retrieved from https://www.nhtsa.gov/document/ 2019-recall-annual-report

Peterson, J. R. \& Schneider, H. S. (2014). Adverse selection in the used-car market: Evidence from purchase and repair patterns in the consumer expenditure survey. The RAND Journal of Economics, 45(1), 140-154.

Peterson, J. R. \& Schneider, H. S. (2017). Beautiful lemons: Adverse selection in durable-goods markets with sorting. Management Science, 63(9), 3111-3127.

RDW. (2018, July 27). Stuiveling open data award [RDW open data]. Retrieved from https:// groups.google.com/d/msg/voertuigen-open-data/wW28nOGABho/wiGOpzUGFAAJ

RDW. (2020). Recalling vehicles and vehicle parts. Retrieved from https://www.rdw.nl/overrdw/information-in-english/vehicle-safety

Rhee, M. \& Haunschild, P. R. (2006). The liability of good reputation: A study of product recalls in the u.s. automobile industry. Organization Science, 17(1), 101-117. 
Seira, E., Elizondo, A., \& Laguna-Müggenburg, E. (2017). Are information disclosures effective? evidence from the credit card market. American Economic Journal: Economic Policy, 9(1), $277-307$.

Strittmatter, A. \& Lechner, M. (2020). Sorting on the used-car market after the volkswagen emission scandal. Journal of Environmental Economics and Management, 101.

Tadelis, S. \& Zettelmeyer, F. (2015). Information disclosure as a matching mechanism: Theory and evidence from a field experiment. American Economic Review, 105(2), 886-905.

Valukas, A. R. (2014). Report to the board of directors of general motors company regarding ignition switch recalls. Jenner\&Block. 


\section{Appendix - For Online Publication}

\section{A Additional statistics about the vehicle market and recalls in the Netherlands}

Figure A.1 shows the market share of the top 20 brands in Netherlands through our sample period. About six manufacturers (Volkswagen, Opel, Peugeot, Renault, Ford and Toyota) have market shares exceeding $6 \%$ while the remaining top 20 brands are primarily Asian and European, with individual market shares between $1 \%$ and $5 \%$.

Figure A.1: Market share by manufacturer, top 20 brands

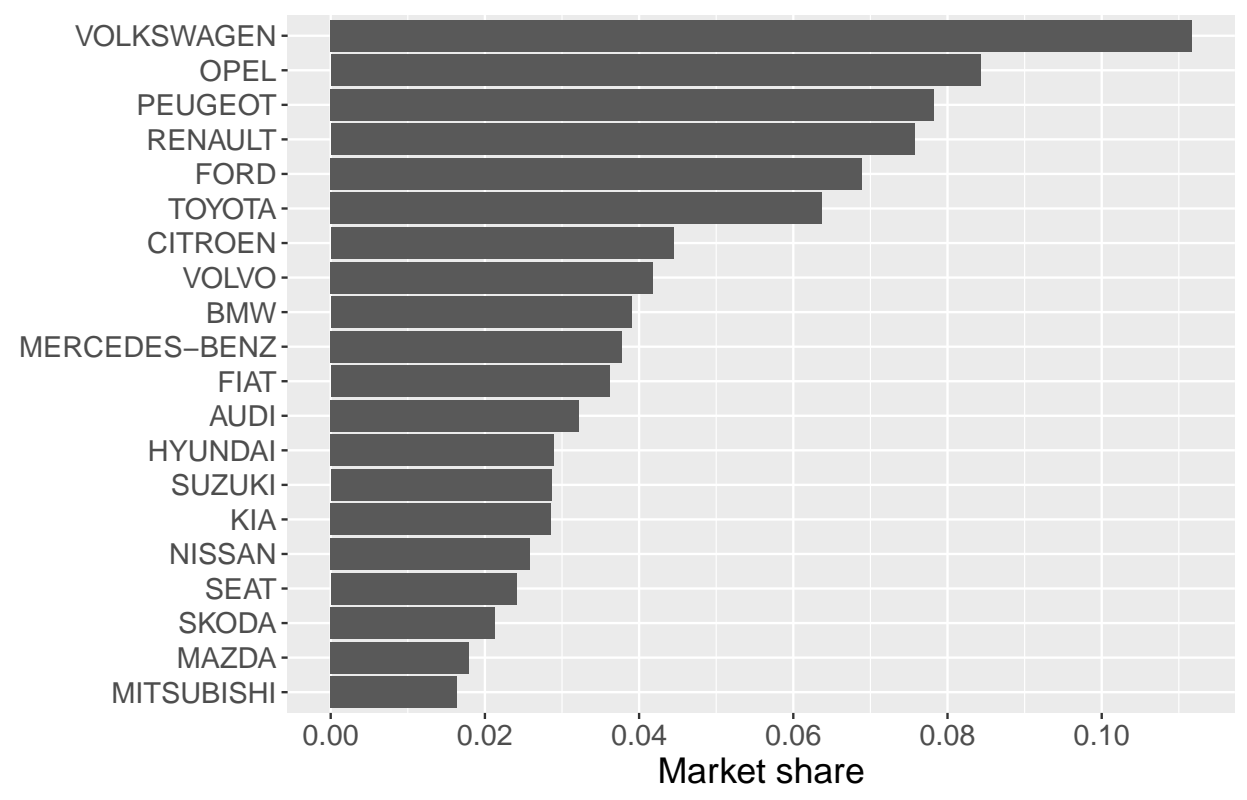

The nature and severity of recalls in the Netherlands is quite heterogeneous. Based on the risk category assigned by RDW, the share of recalls classified as low risk recalls is negligible; little under 40 percent is considered average risk, and the remainder as serious risk. Regarding vehicle parts we observe that more than a third of recalled vehicles are recalled due to problems with safety equipment, primarily airbags and safety belts. Other common causes of recalls are issues concerning brakes, doors, engine, seats, and electrical components. 
There is also considerable variation in the total number of vehicles affected by a specific recall. Figure A.2 shows the distribution of the vehicles involved in recalls, expressed on a log scale. About half of recalls involved fewer than 100 vehicles each in Netherlands, and fewer than 10,000 vehicles worldwide. Still, our data also includes recalls that involved a large number of vehicles both in Netherlands $(>10,000)$, and worldwide $(>1$ million).

Figure A.2: Vehicle recalls by number of vehicles affected (log scale)

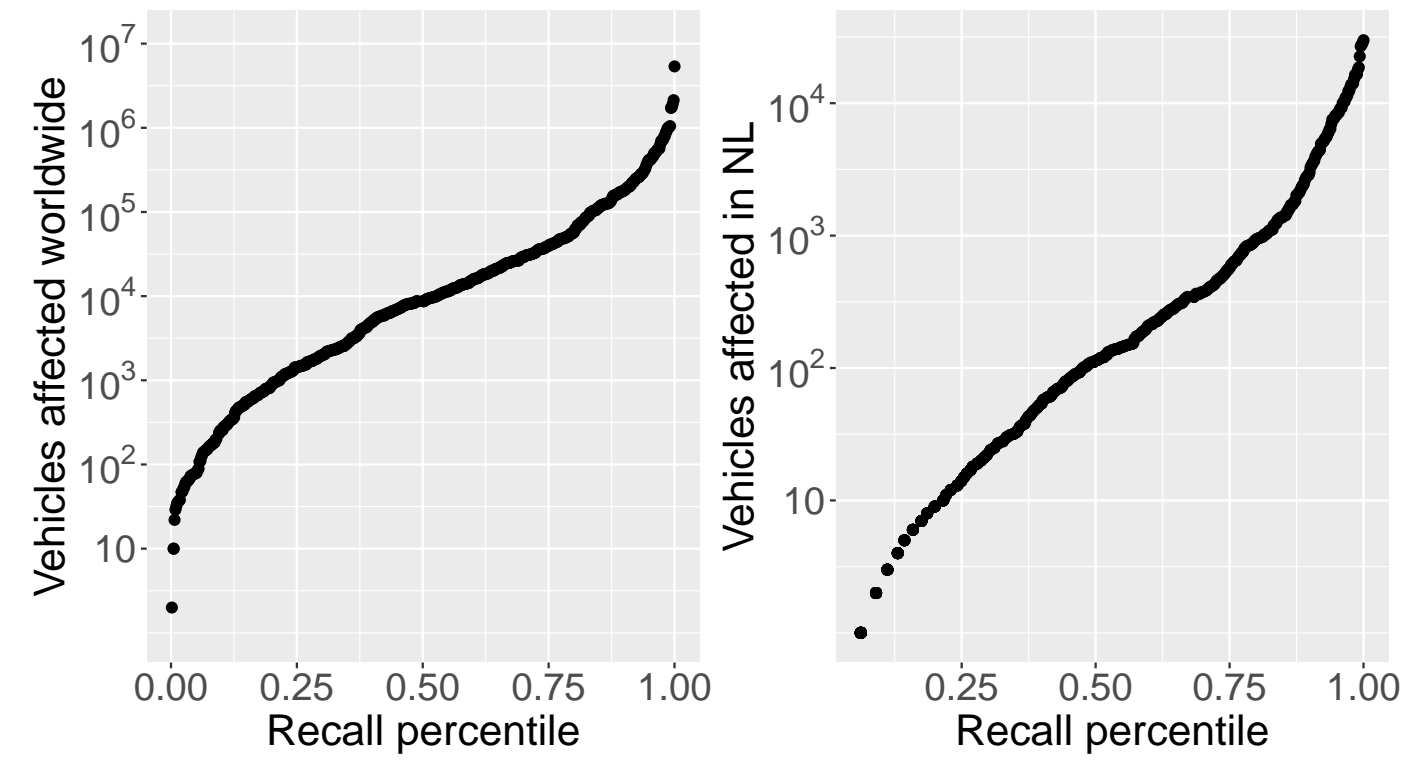

\section{B The effect of recalls on the used vehicle price; an example with two vehicle types}

In this Appendix we use our theoretical framework to solve for the equilibrium supply on the used vehicle market, and derive the effect of the recall on the used vehicle price, conditional on a vehicle transaction taking place. We find that depending on parameter values, this effect could be either positive or negative (see Proposition B.1).

Consider the framework presented in Section 3 and suppose that $c$ can only take on one of two values, low or high: $c \in\left\{c_{L}, c_{H}\right\}$, with $c_{L}<c_{H}$. Suppose also that for all non-recalled vehicles, $c=c_{H}$ for a share $\alpha<1$ of all vehicles, and $c=c_{L}$ for the remainder. Similarly, for all recalled 
vehicles, a share $(1-\gamma) \alpha$ has high $c=c_{H}$ while a share $1-(1-\gamma) \alpha$ has low $c=c_{L}$. We assume that $\gamma \in[0,1]$; recalls are associated lower vehicle quality whenever $\gamma>0$.

For $d$ we make no additional assumptions over and above those discussed above: we assume there exists a weakly negative correlation between the recall signal and the realisation of $d$, such that $D(\mathrm{I}=0) \geq D(\mathrm{I}=1)$.

From here, we use (4) to establish that for $c=c_{L}$, owners will sell their vehicles whenever

$$
b \leq \tilde{b}^{L}(\mathbf{I}) \equiv \frac{V-p^{n}}{\theta_{H}-\theta_{L}}-\left[c_{L}+D(\mathbf{I})\right]
$$

Similarly, if $c=c_{H}$, owners will sell if the following condition is satisfied:

$$
b \leq \tilde{b}^{H}(\mathbf{I}) \equiv \frac{V-p^{n}}{\theta_{H}-\theta_{L}}-\left[c_{L}+D(\mathbf{I})\right]-\left[\theta_{H}-\theta_{L} \alpha(1-\gamma \mathbf{I})\right] \frac{c_{H}-c_{L}}{\theta_{H}-\theta_{L}}
$$

One can straightforwardly observe that $\tilde{b}^{H}<\tilde{b}^{L}$. From here it then follows that if the vehicle is of high observable quality $b>\tilde{b}^{L}$, the vehicle will not be offered on the used car market. If instead $b$ is moderate, such that $\tilde{b}^{H}<b \leq \tilde{b}^{L}$, only vehicle owners with a low realisation of $c$ will be willing to sell their vehicles, while for $b$ equal to or below $\tilde{b}^{H}$, the vehicle will be offered irrespective of the realisation of $c$. Consequently, upon observing that a moderate $b$ vehicle is offered on the market, the buyer will correctly infer that $c$ must equal $c_{L}$.

The situation is different if $b$ is low, as in this case both high and low $c$ vehicles will be offered on the market. As buyers cannot distinguish between these vehicles, their expected value of $c$ will equal $C(b, \mathrm{I})=(1-\gamma \mathbf{I}) \alpha c_{H}+(1-(1-\gamma \mathbf{I}) \alpha) c_{L}$, with $\mathrm{I}=1$ if the vehicle has been subject to a recall, and $\mathrm{I}=0$ otherwise. As in this case, $c_{H}<C(b, \mathrm{I})$, the information asymmetry will imply that the owner of a $c=c_{H}$ vehicle is unable to recoop the full value of his vehicle.

The recall then affects the vehicle supply through the two channels discussed in the main text. First, a downward adjustment in $D(\mathrm{I})$ following a recall will make both high and low $c$ owners more inclined to sell: from $(\mathrm{B} .1)$ and $(\overline{\mathrm{B} .2})$, both $\tilde{b}^{L}$ and $\tilde{b}^{H}$ are decreasing in $D(\mathrm{I})$. Second, insofar recalled vehicles are more likely of low quality $c$, which is the case whenever $\gamma>0$, the increase 
in the information may cause the high quality $c$ owner to refrain from selling her vehicle.

In turn, by (3), (B.1) and (B.2) we obtain the following solution for the used vehicle price:

$$
p^{u}= \begin{cases}\theta_{L}\left[b+c_{L}+D(\mathbf{I})\right] & \text { if } b>\tilde{b}^{H}(\mathbf{I}) \\ \theta_{L}\left[b+c_{L}+D(\mathbf{I})+(1-\gamma \mathbf{I}) \alpha\left(c_{H}-c_{L}\right)\right] & \text { if } b \leq \tilde{b}^{H}(\mathbf{I})\end{cases}
$$

We can then establish under what conditions the recall leads to an reduction in the resale value of the recalled vehicles, and when the resale value may in fact increase:

Proposition B.1. If $\alpha(1-\gamma)\left(c_{H}-c_{L}\right)>D(0)-D(1)>\frac{\theta_{L}}{\theta_{H}-\theta_{L}} \alpha \gamma\left(c_{H}-c_{L}\right)$, then the recall may either increase or reduce $p^{u}$. Otherwise, the recall will weakly reduce $p^{u}$.

Proof. First suppose that $b>\tilde{b}^{H}(0)$ and $b>\tilde{b}^{H}(1)$. Then from $(\overline{\mathrm{B} .3}), p^{u}=\theta_{L}\left[b+c_{L}+D(\mathrm{I})\right]$ and by $D(0) \geq D(1)$, the recall weakly reduces $p^{u} \cdot p^{u}$ is strictly reduces if $D(0)>D(1)$.

Next suppose that $b \leq \tilde{b}^{H}(0)$ and $b \leq \tilde{b}^{H}(1)$. Then $p^{u}=$ $\theta_{L}\left[b+c_{L}+D(\mathrm{I})+(1-\gamma \mathbf{I}) \alpha\left(c_{H}-c_{L}\right)\right]$ and by $D(0) \geq D(1), c_{H}>c_{L}$ and $\alpha \geq 0$, the recall (weakly) reduces $p^{u}$.

In turn suppose that $\tilde{b}^{H}(0) \geq b>\tilde{b}^{H}(1)$, such that prior to the recall, $p^{u}=$ $\theta_{L}\left[b+c_{L}+D(0)+\alpha\left(c_{H}-c_{L}\right)\right]$ while after the recall $p^{u}=\theta_{L}\left[b+c_{L}+D(1)\right]$. From $(\underline{\mathrm{B} .2})$, this situation may occur if $\theta_{L} \alpha \gamma \frac{c_{H}-c_{L}}{\theta_{H}-\theta_{L}}>D(0)-D(1)$. Then the recall weakly reduces $p^{u}$ whenever $\theta_{L}\left[b+c_{L}+D(0)+\alpha\left(c_{H}-c_{L}\right)\right] \geq \theta_{L}\left[b+c_{L}+D(1)\right]$, which requires $D(0)-D(1)+$ $\alpha\left(c_{H}-c_{L}\right) \geq 0$. As $D(0) \geq D(1), c_{H}>c_{L}$ and $\alpha \geq 0$ this assumption is always satisfied.

Finally suppose that $\tilde{b}^{H}(1) \geq b>\tilde{b}^{H}(0)$, such that prior to the recall, $p^{u}=\theta_{L}\left[b+c_{L}+D(0)\right]$ while after the recall $p^{u}=\theta_{L}\left[b+c_{L}+D(1)+(1-\gamma) \alpha\left(c_{H}-c_{L}\right)\right]$. From (B.2), this situation may occur if $\theta_{L} \alpha \gamma \frac{c_{H}-c_{L}}{\theta_{H}-\theta_{L}}<D(0)-D(1)$. Then the recall weakly reduces the price whenever $\theta_{L}\left[b+c_{L}+D(0)\right] \geq \theta_{L}\left[b+c_{L}+D(1)+\alpha(1-\gamma)\left(c_{H}-c_{L}\right)\right]$, which requires $D(0)-D(1) \geq$ $\alpha(1-\gamma)\left(c_{H}-c_{L}\right)$. Conversely, the price increases following the recall if $D(0)-D(1)<$ $\alpha(1-\gamma)\left(c_{H}-c_{L}\right)$. 


\section{Vehicle repair results}

The parallel trend test illustrated in Figure 6 shows that following a recall, vehicle resale rates are not constant over time. Rather, for the first months after the recall, resales fall, while they increase afterwards.

A potential explanation for this time pattern of resales may be vehicle repairs. When a vehicle is recalled, vehicle owners are offered to repair their vehicles free of charge. Even though there is no restriction on resales of recalled but unrepaired vehicles, and the right to a repair does not expire, vehicle owners may prefer to have their vehicles repaired prior to the resale for several reasons. First, the seller's decision to not repair a recalled vehicle prior to resale may be perceived by the buyer as a signal for a lack of attention to the vehicle condition in general. Therefore, the buyer may worry it is harder to sell the vehicle in the absence of a repair. Second, while history on past recalls is accessible through several websites, many online resources, including the RDW own database, only report open (unrepaired) recalls. As such, buyers may be less likely aware of the recall after the vehicle has been fixed. As it may take some time before vehicle owners can make an appointment for such a repair, one could argue that the initially negative and then positive effect of the recall is due to delays in vehicle repairs.

Figure C.1 shows the cumulative share of recalled vehicles that have been repaired over time (as reported by RDW). We include only recalls initiated after the beginning of our sampling period. With the exception of recalls originating in November 2017, recalled vehicles generally share a similar evolution in terms of fixing: on average, about 75 percent of the vehicles are fixed after 6-7 months. Repairs fully level off about one year after the recall, leaving approximately 1 of 8 vehicles unrepaired.

To assess the role of repairs in resale decisions over time, we use information on vehicle repairs, and the following modification of equation (7): 
Figure C.1: Share of repaired vehicles over time

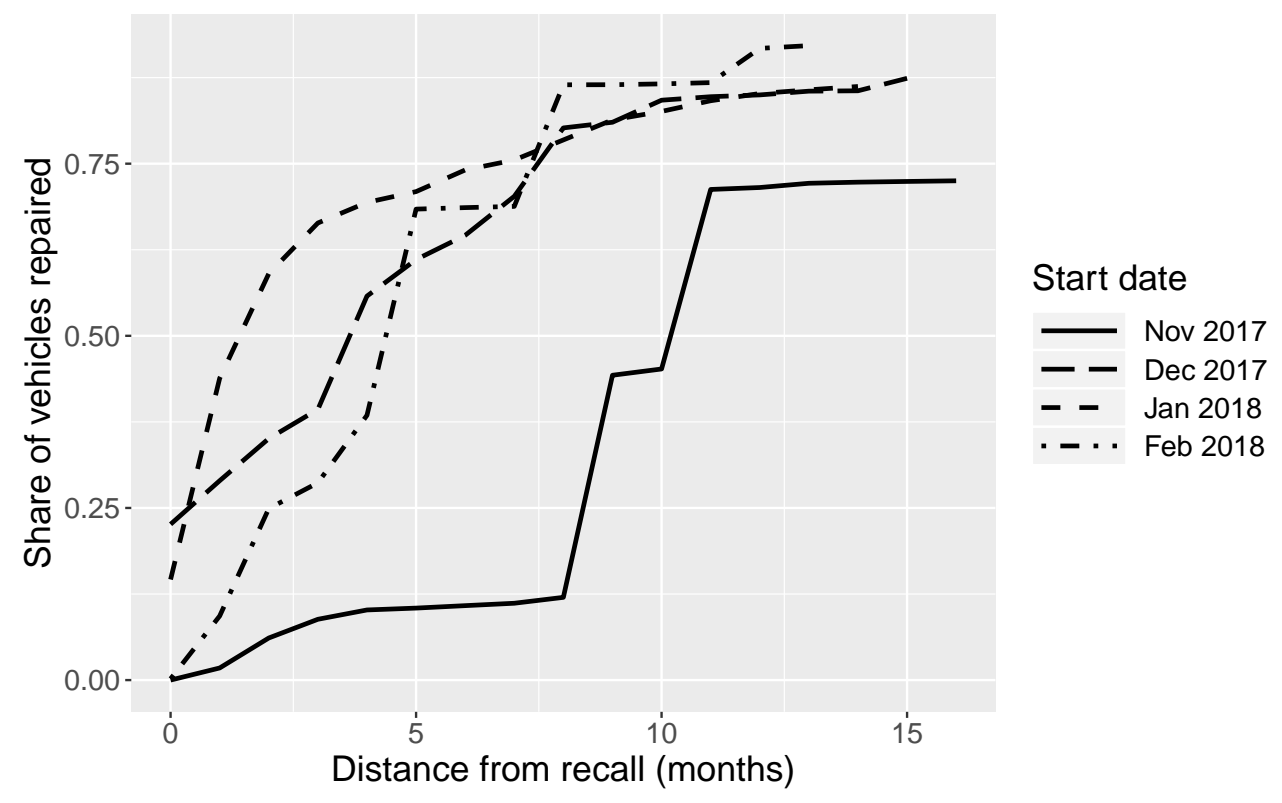

$$
\begin{aligned}
\text { Resold }_{i j r t}= & \alpha+\sum_{d<0} \beta_{d} \text { Recalled }_{i} * \text { Tt }_{d}+\sum_{d \geq 0} \beta_{d} \text { Recalled }_{i} * \text { Unfixed }_{i d} * \text { Tt }_{d}+ \\
& +\sum_{d \geq 0} \beta_{d} \text { Recalled }_{i} * \text { Fixed }_{i d} * \text { Tt }_{d}+\delta \text { Age }_{i t}+\text { SDSale }_{i t}+\phi_{j r}+ \\
& +\psi_{j t}+u_{i j r t},
\end{aligned}
$$

where $U n$ fixed $_{i d}$ is an indicator on whether the recalled car $i$ remained unrepaired in period $d$, and Fixed $d_{i d}$ is an indicator on whether the car was repaired during, or prior to, this period.

Because the decision of fixing the car or not is made by the owner, we face a potential selfselection problem: certain owners might be keen to get their car repaired, or to get it repaired faster, than others. This could be owners who are particularly interested in selling their vehicles, or alternatively owners who place a high value on their car and prefer to continue using it. Hence, in the absence of a reliable instrument correlated with the timing of the decision to fix the vehicle, the effect of fixing on the vehicle on resales cannot be established causally. As such, our analysis serves an illustrative purpose, rather than a causal one. 
Our results are presented in Figure C.2. In the figure, resale rates of unrepaired and repaired recalled vehicles generally follow a similar trend over time across all specifications. The resale likelihood is generally lower for repaired vehicles, albeit not significantly so. An exception are resales 1-3 months and 4-6 months after the recall, which in the specifications including only version FE or version-recall FE are significantly lower for vehicles that have received a repair than for those vehicles that have not ${ }^{45}$ All in all, this shows that, in the short run, repairing the vehicle is associated with a lower resale probability. As noted above this can be explained by self selection, where owners who fix their cars, or who fix it sooner, do so because they value their vehicles and want to continue to drive them. As such, the results above should be interpreted as a lack of evidence in favor of, rather than evidence against, the hypothesis that the time pattern of resales following a recall is due to delays in repair.

\footnotetext{
${ }^{45}$ This lower and significant resale probability for fixed vehicles also prevails when considering the effect across all periods as in equation (5).
} 
Figure C.2: Recalls and resales over time, by repair status.
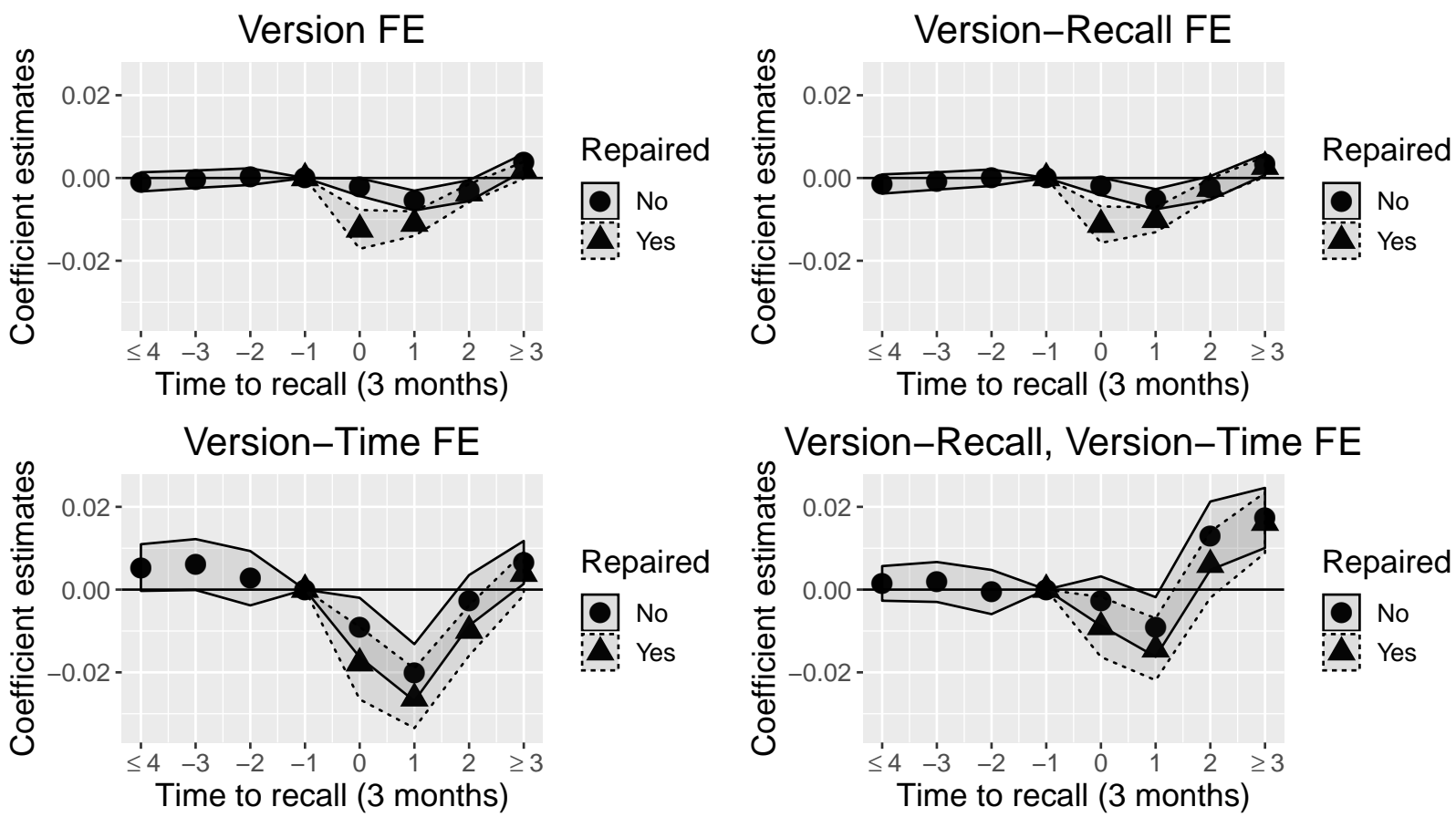

Notes: Results from equation (C.1). The horizontal axis shows the time (in units of three months) since the start of the recall (time 0). Each coefficient represents the change in probability of a resale occurring during each three month period, by repair status. Time period -1 is the omitted coefficient. The four panels show the results from using different groups of fixed effects. Standard errors at the vehicle type-time level. The shadowed areas represent $95 \%$ confidence intervals.

\section{Effect of recalls on the depreciation factor; heterogeneity}

\section{across market segments}

In this section we assess whether the effect of recalls on prices is heterogeneous across market segments. As in the main text, we use the depreciation factor as a proxy for prices. The estimation follows equation (8) and, where we again run separate regressions for each of the two market segments we split the sample in to. Results are presented in tables D.1, D.2 and D.3, and show no systematic heterogeneity in the effects of recalls on the depreciation factor across market segments. 


\begin{tabular}{l|cccc} 
& \multicolumn{5}{|c}{ Panel A: Price $<30 \mathrm{~K}$ EUR } \\
& (i) & (ii) & (iii) & (iv) \\
\hline RecalledPost & 0.0008 & $0.0038^{* *}$ & $-0.0071^{* *}$ & 0.0002 \\
Recalled & $(0.0018)$ & $(0.0019)$ & $(0.0034)$ & $(0.0041)$ \\
& -0.0032 & & 0.0052 & \\
& $(0.0027)$ & & $(0.0035)$ & \\
& \multicolumn{5}{|c}{ Panel B: Price $\geq 30 \mathrm{~K}$ EUR } \\
& $(\mathrm{i})$ & (ii) & $(\mathrm{iii})$ & $(\mathrm{iv})$ \\
\hline RecalledPost & -0.0018 & 0.0003 & -0.0024 & 0.0051 \\
& $(0.0023)$ & $(0.0023)$ & $(0.0065)$ & $(0.0084)$ \\
Recalled & $0.0062^{* *}$ & & 0.0081 & \\
& $(0.0028)$ & & $(0.0064)$ & \\
& \multicolumn{5}{c}{ Yo } \\
Version-Recall FE & No & Yes & Yes \\
Version-Time FE & No & No & Yes & Yes
\end{tabular}

Table D.1: Depreciation factor results by real vehicle listing price, separate regressions

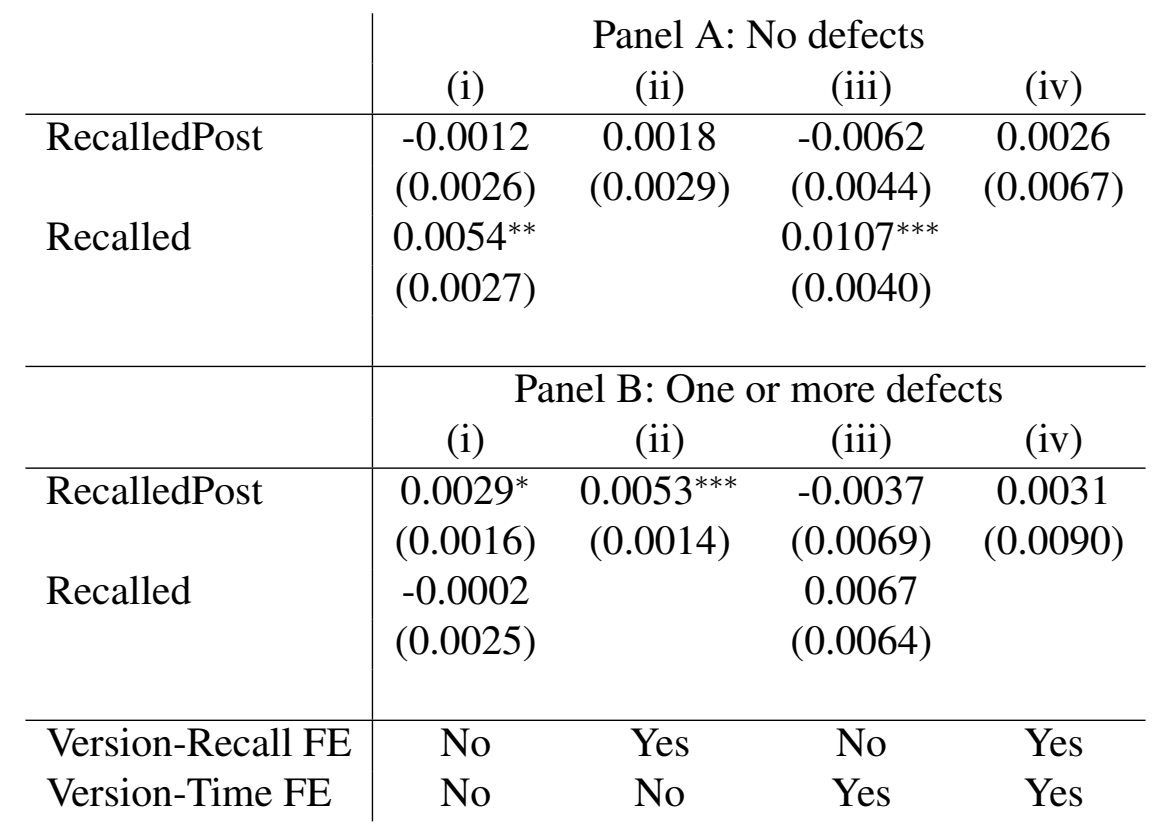

Table D.2: Depreciation factor results by inspection defects, separate regressions 


\begin{tabular}{|c|c|c|c|c|}
\hline & \multicolumn{4}{|c|}{ Panel A: Brand rating below 7} \\
\hline & (i) & (ii) & (iii) & (iv) \\
\hline \multirow[t]{2}{*}{ RecalledPost } & 0.0001 & 0.0034 & -0.0062 & 0.0020 \\
\hline & $(0.0022)$ & $(0.0026)$ & $(0.0026)$ & $(0.0052)$ \\
\hline \multirow{4}{*}{ Recalled } & 0.0024 & & $0.0068^{*}$ & \\
\hline & $(0.0025)$ & & $(0.0038)$ & \\
\hline & \multicolumn{4}{|c|}{ Panel B: Brand rating equal or above 7} \\
\hline & (i) & (ii) & (iii) & (iv) \\
\hline \multirow[t]{2}{*}{ RecalledPost } & -0.0018 & 0.0010 & -0.0045 & 0.0032 \\
\hline & $(0.0024)$ & $(0.0024)$ & $(0.0045)$ & $(0.0046)$ \\
\hline \multirow[t]{2}{*}{ Recalled } & 0.0014 & & 0.0057 & \\
\hline & $(0.0036)$ & & $(0.0048)$ & \\
\hline Version-Recall FE & No & Yes & No & Yes \\
\hline Version-Time FE & No & No & Yes & Yes \\
\hline
\end{tabular}

Table D.3: Depreciation factor results by brand reputation, separate regressions 